\title{
A. M. Mathai Centre for Mathematical and Statistical Sciences: A Brief History of the Centre and Prof. Dr. A. M. Mathai's Research and Education Programs at the Occasion of His 85th Anniversary
}

\author{
Hans J. Haubold \\ Office for Outer Space Affairs, United Nations Vienna International Centre, Vienna, Austria \\ Email: hans.haubold@gmail.com
}

How to cite this paper: Haubold, H. J. (2020). A. M. Mathai Centre for Mathematical and Statistical Sciences: A Brief History of the Centre and Prof. Dr. A. M. Mathai's Research and Education Programs at the Occasion of His 85th Anniversary. Creative Education, 11, 356-405.

https://doi.org/10.4236/ce.2020.113028

Received: February 12, 2020

Accepted: March 17, 2020

Published: March 20, 2020

Copyright $\odot 2020$ by author(s) and Scientific Research Publishing Inc. This work is licensed under the Creative Commons Attribution International License (CC BY 4.0).

http://creativecommons.org/licenses/by/4.0/

\section{(c) (i) Open Access}

\begin{abstract}
A brief history of the Centre for Mathematical and Statistical Sciences, Kerala, India, is given and an overview of Mathai's research and education programs in the following topics is outlined: Fractional Calculus; Functions of Matrix Argument-M-transforms, M-convolutions; Krätzel integrals; Pathway Models; Geometrical Probabilities; Astrophysics-reaction rate theory, solar neutrinos; Special Functions-G and H-functions; Multivariate Analysis; Algorithms for Non-linear Least Squares; Characterizations-characterizations of densities, information measure, axiomatic definitions, pseudo analytic functions of matrix argument and characterization of the normal probability law; Mathai's Entropy-entropy optimization; Analysis of Variance; Population Problems and Social Sciences; Quadratic and Bilinear Forms; Linear Algebra; Probability and Statistics.
\end{abstract}

\section{Keywords}

Fractional Calculus, Functions of Matrix Argument, Special Functions of Mathematical Physics, Geometrical Probabilities, Multivariate Analysis, Characterization, Algorithms, Quadratic and Bilinear Forms, Entropy, Reaction, Diffusion, Solar Neutrinos

The Centre for Mathematical Sciences (CMS) was established in 1977 and registered in Trivandrum, Kerala, India, as a non-profit scientific society under the 
Travancore-Cochin Literary, Scientific and Charitable Societies Registration Act XII of 1955, and as a research and education centre covering all aspects of mathematics, statistics, mathematical physics, computer and information sciences. Applicants for incorporation were K.T. Chandy, A. Abraham, J. Zachariah, V.I. Subramoniam, K. Sankara Rao, Y. Sitaraman, and A. George, with Professor George taking the lead.

\section{Research and Education in Mathematical and Statistical Sciences}

Initial activities of research concentrated on population studies and applied statistics, the areas of interest of Professor George. Research and project activities started at CMS and at one time there were 35 project staff. CMS has executed a large number of research and education projects for central and state governmental agencies.

By the end of 1984 Professor George passed away and then governing council of CMS requested A. M. Mathai to take over and build it up into an international centre of excellence. Professor Mathai agreed to volunteer for five years, from 1985 to 1990, and build it up into an international centre of excellence, provided support came from the State Government in the form of basic building and ground. All the senior scientists in mathematical sciences in Kerala gave moral support to CMS and volunteered in running research and education programs at CMS (Figure 1).

In 2002, CMS has shifted its office and library into St Thomas College Pala compound into a one-story building donated by the Diocese of Palai, Kerala, India. From 2006 CMS became a Department of Science and Technology (DST), Government of India, Centre for Mathematical Sciences with sufficient financial support from DST. Then CMS could recruit up to $18 \mathrm{PhD}$ students and a core faculty of five. Full-fledged activities of research, education at the undergraduate and research level started, conferences, workshops and lecture series by leading researchers around the world initiated. By this time CMS had become a research

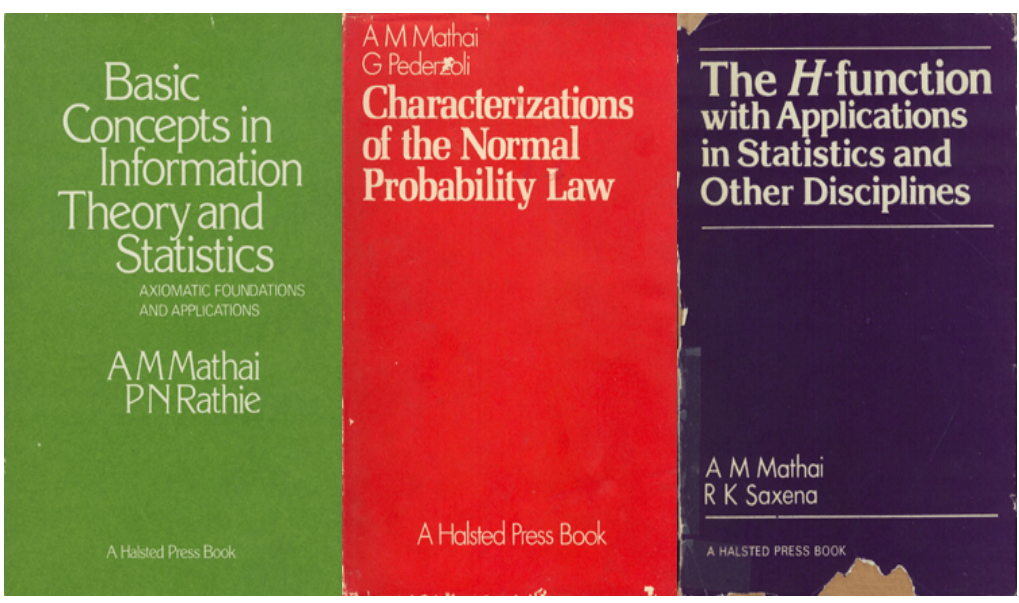

Figure 1. A. M. Mathai's Research Program in mathematics, statistics, and physics developed at the Department of Mathematics and Statistics, McGill University, Montreal, Canada, in the 1970's. 
centre of Banaras Hindu University (BHU) in Varanasi, India, Anna University, Chennai, India, and Mahatma Gandhi University, Kottayam, India.

Since 2006, the CMS has grown into a leading research centre of international repute in aspects of mathematical sciences, particularly those concerning research in astrophysics, special functions of mathematical physics, statistics, and fractional calculus. CMS had a publication series (books, proceedings, collections of research papers, lecture notes), a newsletter of two issues per year, a mathematics modules series (self-study books on basic topics) and a mathematical sciences series for the general public

(documents archive available at http://neutrino.aquaphoenix.com/cmsintl.org/).

CMS has been making efforts to keep pace with developments all around the world and making contributions to bring India into the frontline research in mathematical sciences and allied disciplines. In the publications series CMS brought out around 40 publications, two of which were taken as such and published by Springer, New York, one in 2008 (Mathai \& Haubold, 2008; Figure 8) and one in 2010 (Mathai, Saxena, \& Haubold, 2010; Figure 9). Newsletter series continued until 2015. Ten modules were brought out in the modules series. Three of these in the area of linear algebra were combined and published by De Gruyter, Berlin and Boston in 2017 (Mathai \& Haubold, 2017a; Figure 2) and another three in the area of probability and statistics were combined and published by De Gruyter, Berlin and Boston in 2017 (Mathai \& Haubold, 2017b; Figure 3). These two volumes also served as education material for the UN-affiliated Regional Centres for Space Science and Technology Education in

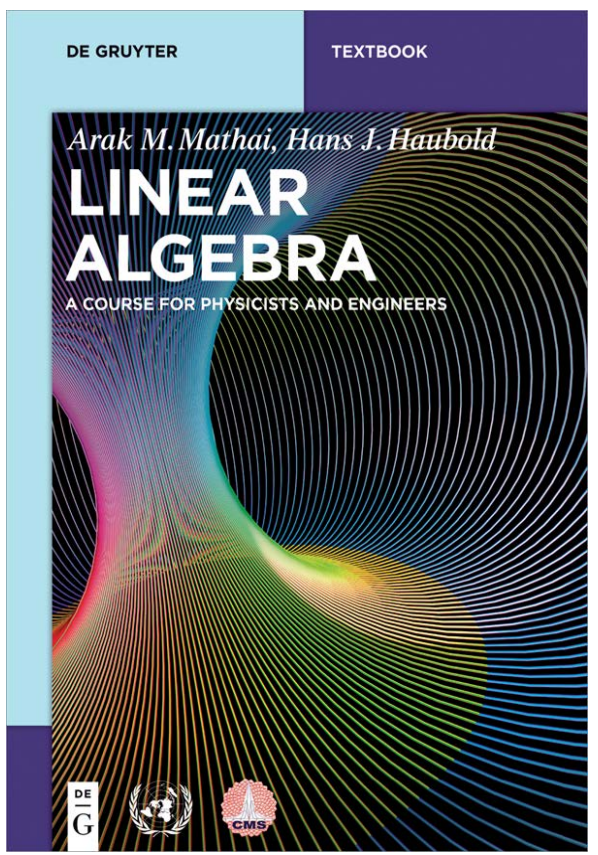

Figure 2. Mathai \& Haubold (2017a): LinearAlgebra for Physicists and Engineers (see also Mathai \& Haubold, 2018a). 


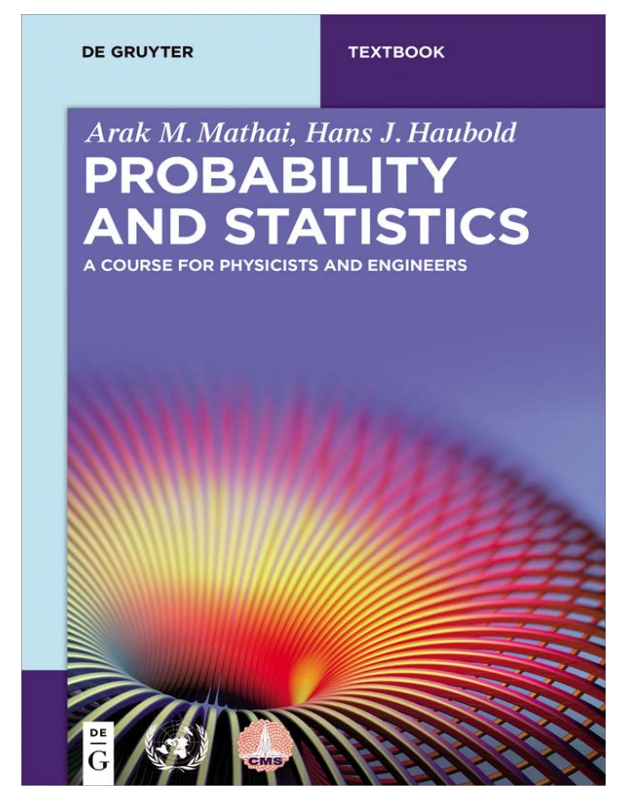

Figure 3. Mathai \& Haubold (2017b): Probability and Statistics for Physicists and Engineers (see also Mathai \& Haubold, 2018a).

Asia and the Pacific, Latin America and the Caribbean, Africa, and Western Asia (Mathai \& Haubold, 2018a).

CMS has been functioning as a national centre for mathematical sciences. The main aim of the centre was to encourage youngsters to do research by organizing activities in mathematical sciences. The research groups at CMS cover major disciplines in mathematical sciences. The following are the research groups at CMS: Astrophysics Research Group, Fractional Calculus Research Group, Special Functions Research Group, Statistical Distribution Theory Research Group, Geometric Probability Research Group, Discrete Mathematics Research Group, Algebra and Analysis Research Group. The research groups at CMS are active in interdisciplinary research.

Astrophysics Research Group

The seniors in the group are HJH and A. M. Mathai (Mathai \& Haubold, 1988, 2018a; Figure 4). R. K. Saxena also joined this group. Senior research fellows in this group are D. Kumar, D. P. Joseph, S. S. Nair, and N. Sebastian. The research group focused on the solar neutrino problem and related issues of thermonuclear reaction rates, closed-form solutions for differential equations governing the internal structure of the Sun, and solar neutrino physics. Experimental data coming from solar neutrino experiments were analysed by Fourier analysis, wavelet analysis and diffusion entropy analysis. Application of special functions of mathematical physics has been encouraged in all research topics.

Fractional Calculus Research Group

The seniors in the group are R.K. Saxena, A. M. Mathai, and HJH (Mathai \& Haubold, 2017c, 2018b, 2018c; Figures 5-7). Senior research fellows working in this group are Seema S. Nair, Anitha Kattuveettil and Nicy Sebastian. Dilip 


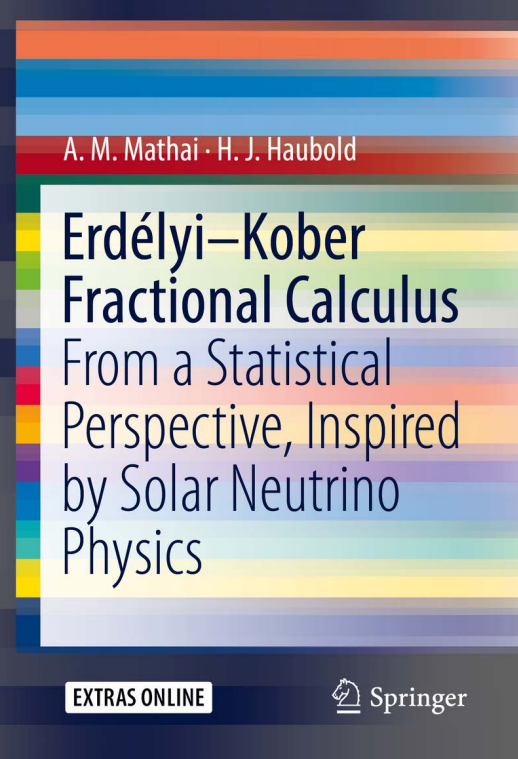

Figure 4. Mathai \& Haubold (2018d): Erdélyi-Kober fractional calculus from a statistical perspective, inspired by solar neutrino physics.

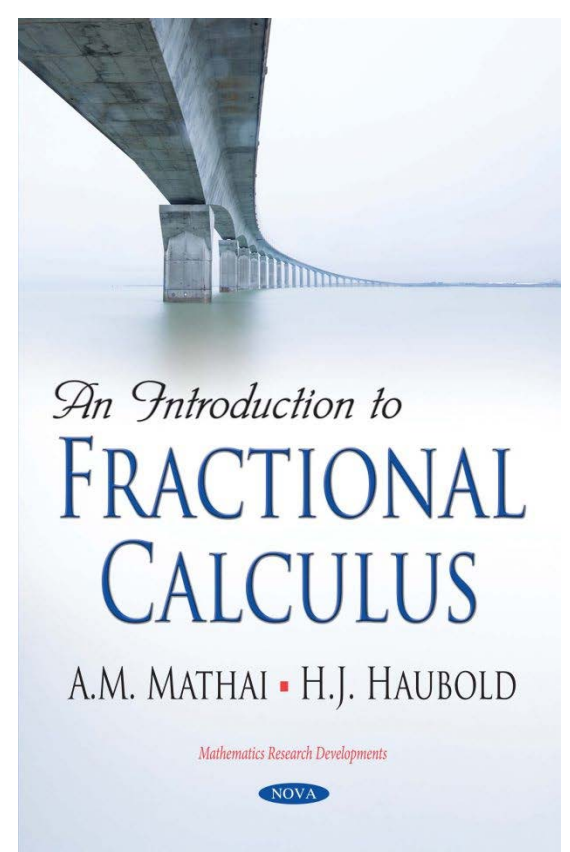

Figure 5. Mathai \& Haubold (2018c): An introduction to fractional calculus.

Kumar also has work in fractional calculus. A national level workshop in this area was held at CMS in November 2009. As an emerging field, CMS conducted several short courses and included fractional calculus in the syllabus of many SERC Schools at CMS. Differentiation and integration are usually regarded as 


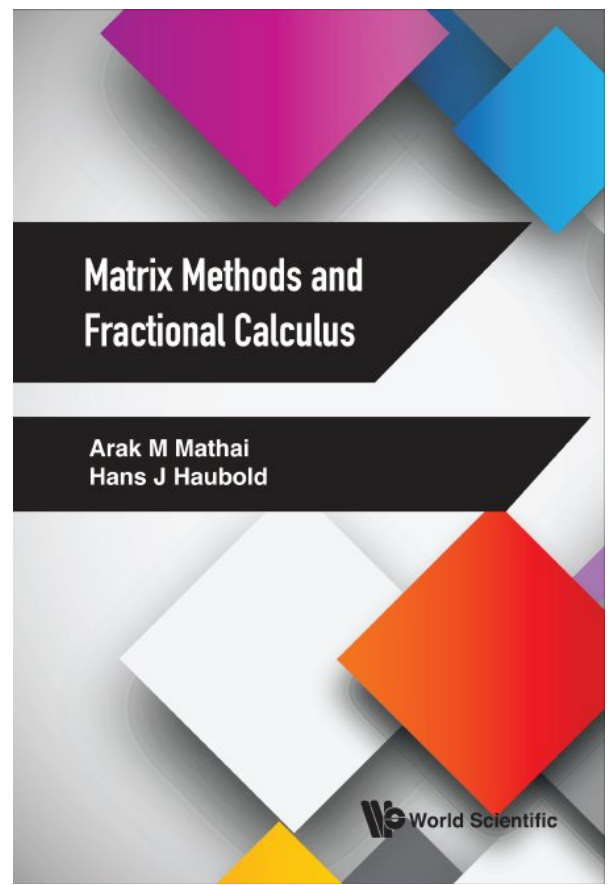

Figure 6. Mathai \& Haubold (2018b): Matrix methods and fractional calculus.

Springer Optimization and Its Applications 122

\section{A.M. Mathai}

H.J. Haubold

\section{Fractional and} Multivariable Calculus

Model Building and Optimization Problems

Figure 7. Mathai \& Haubold (2017c): Fractional and multivariable calculus: Model building and optimization problems.

discrete operations, in the sense that one differentiates or integrates a function once, twice, or any whole number of times. However, in some circumstances it is useful to evaluate a fractional derivative. In a letter to LHospital in 1695, Leibniz 
raised the possibility of generalizing the operation of differentiation to non-integer orders, and LHospital asked what would be the result of half-differentiating $\mathrm{x}$. Leibniz replied It leads to a paradox, from which one day useful consequences will be drawn. The paradoxical aspects are due to the fact that there are several different ways of generalizing the differentiation operator to non-integer powers, leading to inequivalent results. Major contributions in this area from CMS are that fractional calculus was extended to functions of matrix argument in the real and complex domains and extended fractional calculus to the complex domain. Further, research focused on the well established fact that fractional time derivatives do not appear in any established fundamental theory of physics such as statistical mechanics, electrodynamics, or quantum mechanics. Particular attention was placed on the correlation between the diffusion equation and Schroedinger equation and their fractional counterparts.

Special Functions Research Group

The seniors in the group are R.K. Saxena, P. N. Rathie, S. B. Provost, P. Moschopoulos, HJH, and A. M. Mathai. "The H-function: Theory and Applications" (Figure 8) and "Special Functions for Applied Scientists" (Figure 9) are the latest books of this group, which were out from Springer, New York, in 2010 and 2008, respectively. Senior research fellows working in this area are Dilip Kumar, Nicy Sebastian, Dhannya P. Joseph, Seema S. Nair, Anitha Kattuveettil, and Naiju M. Thomas. There are mathematical theories in which some classes of special functions appear naturally. A familiar classification is by increasing complexity, starting with polynomials and algebraic functions and progressing through the elementary transcendental functions etc to the higher transcendental functions. Functions of hypergeometric type can be ordered by the behavior of singular points of the differential equations representing them, or by a group-theoretical analysis of their symmetries. But all these classifications are incomplete. For example, Mathieu functions fall outside the hypergeometric class, and gamma and zeta functions are not the solutions of simple differential equations. For rate and diffusion equations and their fractional generalizations, generalized hypergeometric functions like Meijer's G-function and Fox' $\mathrm{H}$-function need to be made available analytically and numerically. They were of central interest to research and teaching of this research group.

Statistical Distribution Theory Research Group

The seniors in the group are A. M. Mathai, S. B. Provost, W. J. Anderson, HJH, and P. N. Rathie. Generalizations of Dirichlet integrals and Dirichlet densities are introduced by this group. Seemon Thomas, St Thomas College Palai, who is an associate of CMS, has received his $\mathrm{PhD}$ in this area. The pathway model introduced by Mathai in 2005 is popular in statistical distribution theory and non-extensive statistical mechanics. The first $\mathrm{PhD}$ on the pathway model was awarded to Shanoja S. Pai in 2010. Other researchers in this group are Nicy Sebastian, Seema S. Nair, Dhannya P. Joseph, Naiju M. Thomas, Prajitha P. and Princy T. CMS has conducted short-term courses in this area by eminent faculties of national and international standing. Major contributions in this area from 


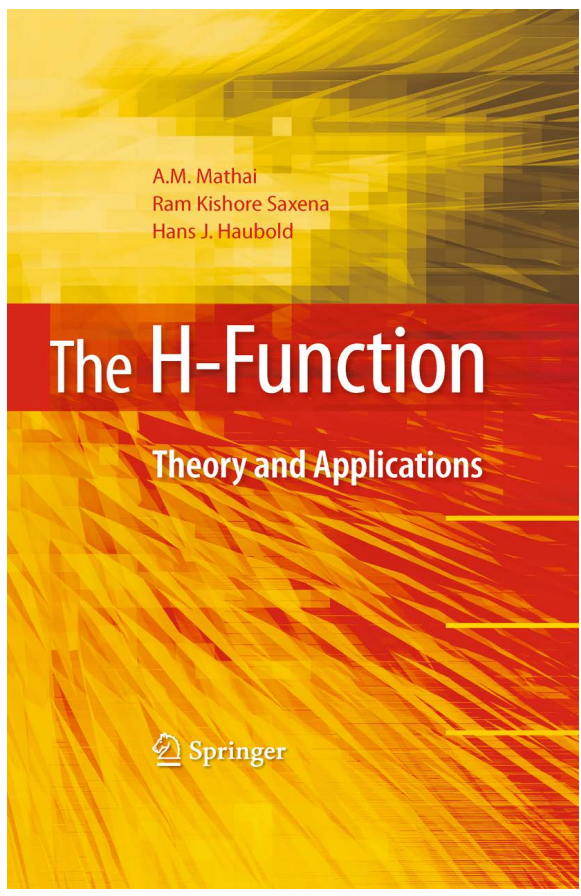

Figure 8. Mathai, Saxena, and Haubold (2010): The h-function: Theory and applications.

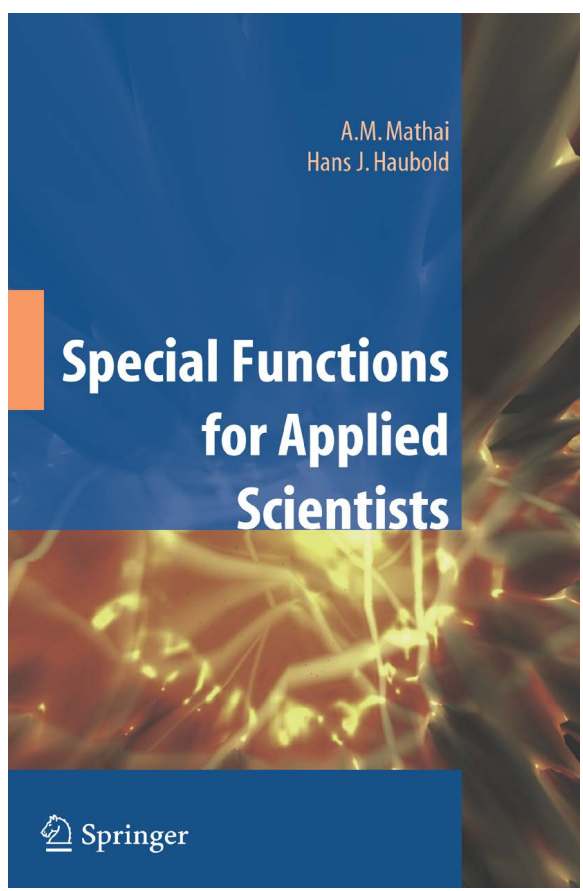

Figure 9. Mathai and Haubold (2008): Special functions for applied scientists.

CMS is that statistical distribution theory is shown to be a powerful tool in interpreting and developing Mellin convolutions of products and ratios, in establishing a connection to fractional integrals, in giving physical interpretations to the concept of M-convolutions introduced in 1970s (Figure 10). 


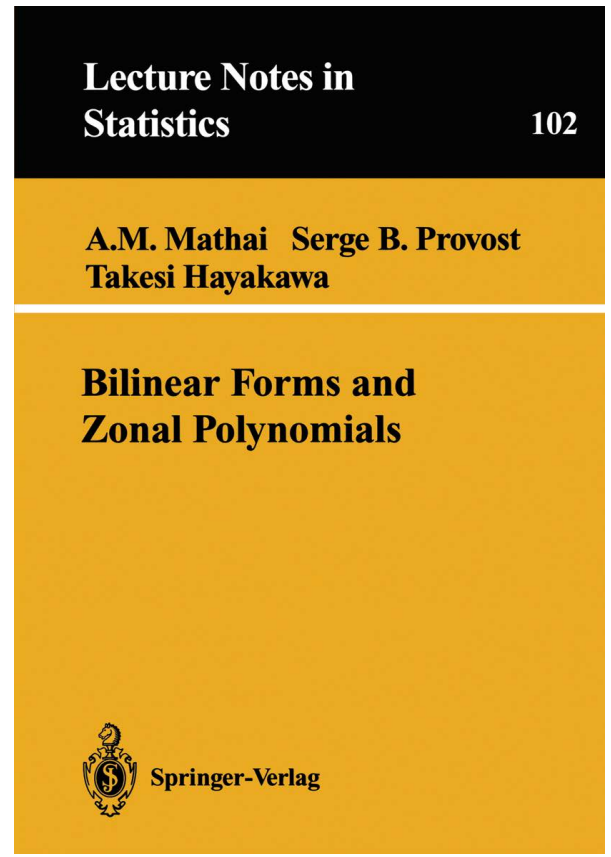

Figure 10. Mathai, Provost, and Hayakawa (1995): Bilinear forms and zonal polynomials.

\section{Geometrical Probability Research Group}

A. M. Mathai is the senior in this group and Seemon Thomas has joined and is working in the group. Major contribution in this area is the replacements of differential and integral geometry techniques with Jacobians of matrix transformations techniques in the study of certain random geometrical configurations and extending the theory to functions of matrix argument (Mathai, 1999a; Figure 12).

Discrete Mathematics Research Group

The seniors in this group are B.D. Acharya (former Advisor to Government of India) who joined as a Visiting Full Professor at CMS, R. Natarajan of Lakehead University, Canada, who was appointed as Reader at CMS, K.A. Germina of the Hill Area Campus of CMS. Two junior research fellows working in this group are Miss Alphy Joseph and Miss Sona Jose. The activities of this group also include building up a strong computer science base at CMS. A national level workshop in this area was held at CMS in February 2010 and another one was conducted in August 2010 in connection with the visit of Professor T. Zaslavsky (USA). Short-term courses are conducted for the enhancement of research in this area.

\section{Algebra and Analysis Research Group}

The seniors in this group are S.C. Mathew and P.G. Romeo. The junior research fellows working in this group are Miss Diana Mary George and Miss Ginu Varghese. Short-term courses are conducted to motivate the students in this area.

Research Recognitions 


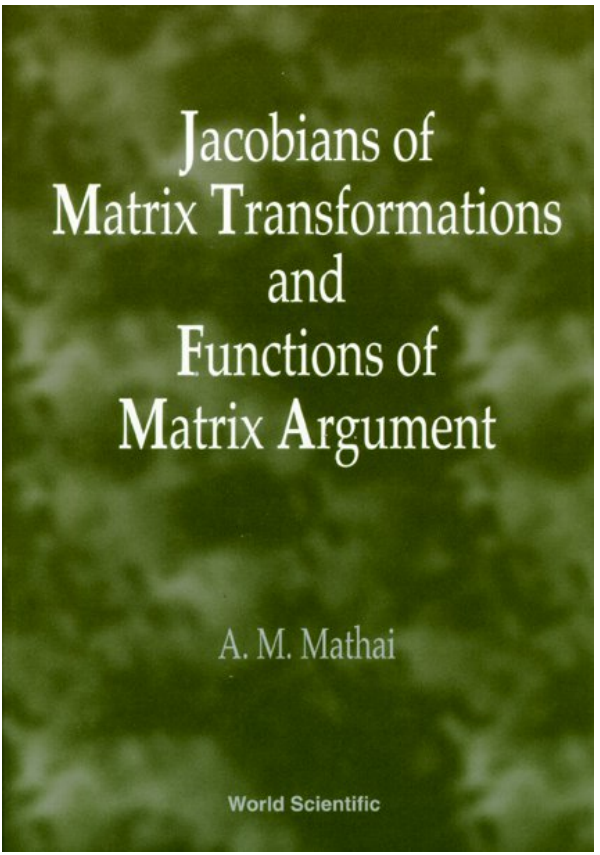

Figure 11. Mathai (1997): jacobians of matrix transfromations and functions of matrix argument.

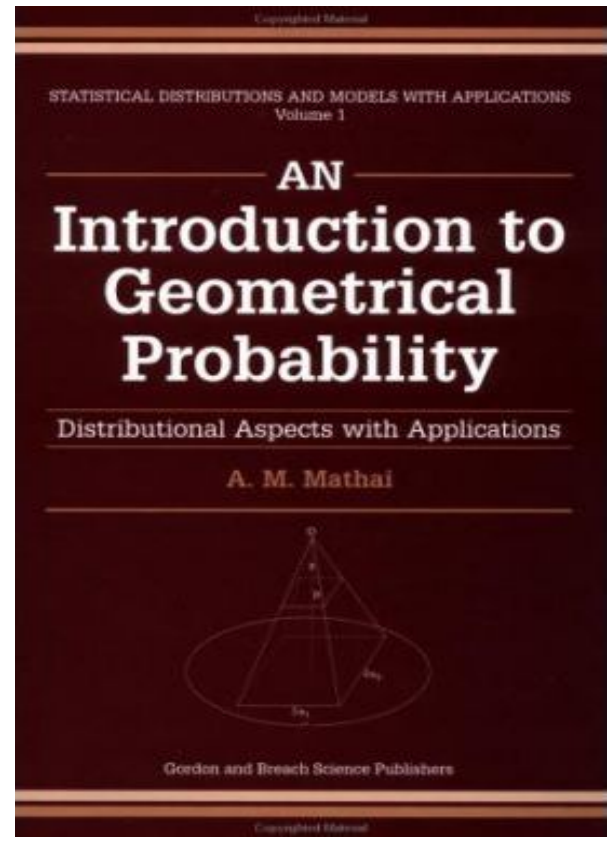

Figure 12. Mathai (1999a): An introduction to geometrical probability: Distributional aspects with applications.

CMS is an approved research centre of three universities, Banaras Hindu University (BHU), Varanasi, Anna University, Coimbatore, and Mahatma Gandhi University (MGU), Kottayam. Students joining CMS for research can register themselves in any of the above universities for $\mathrm{PhD}$ and fulfill the $\mathrm{PhD}$ 
requirements of the respective universities. Some collaborative research is being done with other foreign and national institutions also. Six students from CMS got their $\mathrm{PhDs}$ in the area of statistics from BHU. Three $\mathrm{PhD}$ scholars from CMS received their PhDs from Anna University in the areas of applied analysis, graph theory and fuzzy mathematics. Four PhD scholars from CMS received their PhDs from MGU in the areas of fuzzy mathematics, graph theory and mathematical physics.

Library Development

Back volumes of more than 400 journals (with over 17,000 volumes) and more than 6000 books were available in mathematical sciences for reference purposes at the CMS library. Collections of reprints of individual articles are also arranged in CMS library for ready reference. CMS had built up a good library with books and journals collected by Professor Mathai from Canada and USA.

Portraits Collection

CMS library had a collection of the portraits of over 300 world mathematicians. The enlarged and laminated color portraits were placed on the library walls at CMS for motivating the school/colleges students to do work in mathematical sciences. Students of mathematical sciences have attended lectures about masters in mathematical sciences, they have learnt about the discoveries of these masters and the students had the opportunity to see their portraits and learn more about these great men and women by studying at CMS library.

The summary of achievements of CMS (later CMS was renamed CMSS (Centre for Mathematical and Statistical Sciences)) from 2007 to 2014, when it was a DST Centre, is the following:

Achievements of CMSS 2007-2014

Number of national/international level awards won by CMSS scholars $=19$.

Number of research papers published in international refereed journals \& proceedings $=170$.

Number of PhDs produced by CMSS = 13 [6 from BHU, 4 from MGU, and 3 from Anna University, Chennai].

Number of research level students educated through all-India SERC Schools > 285 (Figure 13).

Number of undergraduate students educated through Undergraduate Mathematics Camps $>1020$.

Number of paper presentations abroad by CMSS research scholars $=8$.

Number of paper presentations in India by CMSS scholars $>65$.

Number of invited and keynote addresses by the faculty of CMSS, including a lead keynote address and 3 keynote addresses at a United Nations' workshops > 63.

Number of conferences sponsored or co-sponsored by CMSS $=7$.

Number of distinguished visitors to CMSS from abroad $>32$.

Number of distinguished national visitors to CMSS $>63$.

Number of international research collaborators with CMSS faculty and scholars $=8$ 


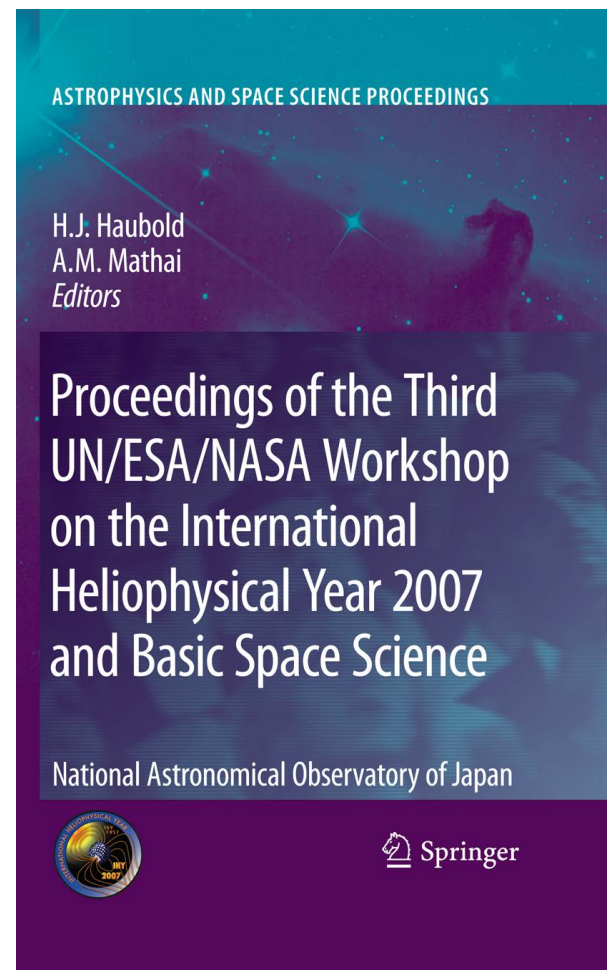

Figure 13. Haubold \& Mathai (2010): Proceedings of the third UN/ESA/NASA Workshop on the international heliophysical year 2007 and basic space science.

Number of national level research collaborators with CMSS faculty and scholars $=7$.

The Honorary Director

Professor A. M. Mathai was honored by several institutions and national societies in India and thrice by the United Nations, once in Tokyo, Japan, once in Daejeon, Korea, and once in Quito, Ecuador and he gave keynote addresses also. He was the President of the Indian Mathematical Society for one term (the oldest professional society in India), current President of the Society for Special Functions and their Applications, Chairman of the Kerala State Statistical Commission (2014-2018), he was given Lifetime Achievement Award and Fellowship by the Indian Society for Probability and Statistics, and he is a Member of the Loka Kerala Sabha.

\section{Achievements in Research and Education by A. M. Mathai}

In this article Arak M. Mathai's research accomplishments in mathematics and statistics are discussed which he achieved and shared with collaborators, postdocs, and students at McGill University, at the Centre for Mathematical and Statistical Sciences, and at annual United Nations workshops over a period of time of more than 60 years (Figure 14, Figure 15).

In the 1970s Mathai and collaborators developed an axiomatic theory providing axiomatic definitions for basic concepts in information theory and statistics. Focus was placed on functional equations, dispersion, principles of statistical 


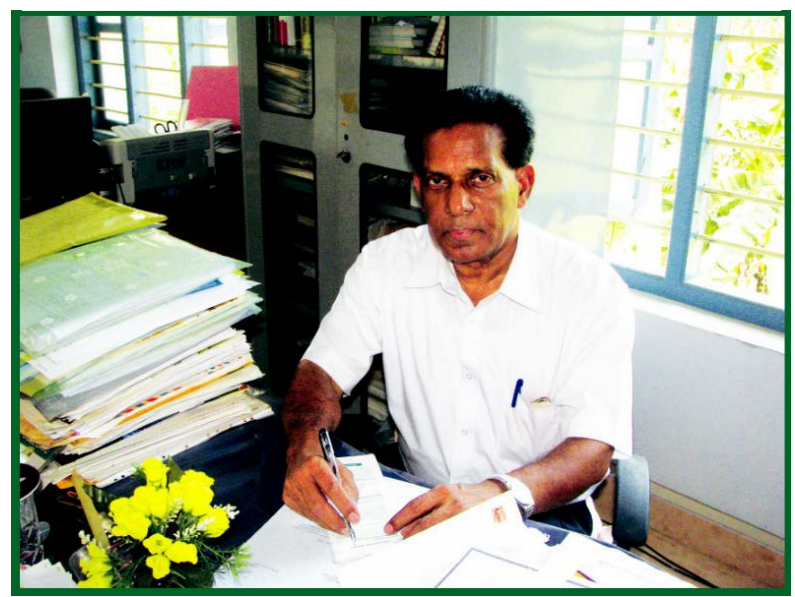

Figure 14. A.M. Mathai.

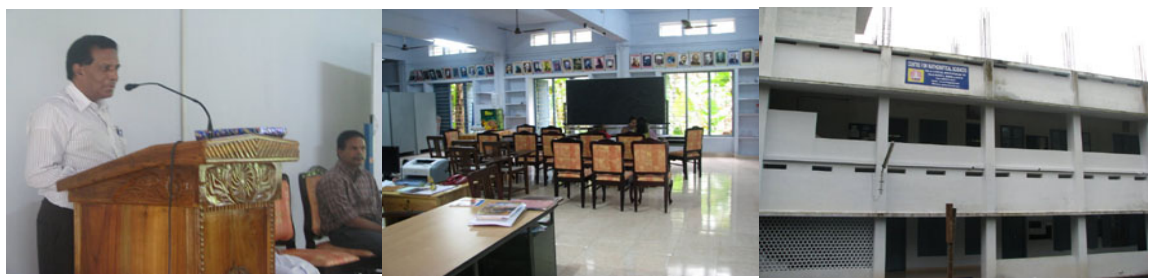

Figure 15. Centre for mathematical and statistical sciences.

inference, and inequalities among information theory and statistical measures. Conditional distributions, characterizations through independence of linear and quadratic statistics and characterizations with the help of differential equations and functional equations were developed in detail. Special functions, persistent in research and education in mathematics, statistics, and natural sciences, today available in the Digital Library of Mathematical Functions and accessible as a free Web-based collection of formulas (http://dlmf.nist.gov), cross-linked and with live graphics that can be magnified and rotated and downloaded became increasingly important in Mathai's research. New results for theory and applications of generalized hypergeometric functions, among them Meijer's G-function and Fox' $\mathrm{H}$-function extended to real scalar functions of matrix argument when the argument matrix is real or in the complex domain, became part of Mathai's research. Eventually the full programme for research and education in these fields of mathematics and statistics with applications to natural sciences was published in a triology of monographs (Mathai \& Rathie, 1975; Mathai \& Pederzoli, 1977; Mathai \& Saxena, 1978; Figure 1). This research and education program set the stage for CMS (Figure 15).

A.M. Mathai was born on 28 April 1935 in Arakulam, near Palai, in the Idukki district of Kerala, India as the eldest son of Aley and Arakaparampil Mathai. After completing his high school education in 1953 from St. Thomas High School, Palai, he joined St. Thomas College, Palai with record marks and obtained his B.Sc. degree in Mathematics in 1957. In 1959, he completed his Master's Degree in Statistics from the University of Kerala, Thiruvananthapuram, Kerala, India; 
he achieved a first class degree, the first rank and a gold medal. Then, he joined St. Thomas College, Palai, University of Kerala, as a Lecturer in Mathematics and served there until 1961. He obtained a Canadian Commonwealth scholarship in 1961 and went to the University of Toronto, Canada, to complete his M. A. degree in Mathematics in 1962. He was awarded a PhD from the University of Toronto, Canada, in 1964. Then, he joined McGill University, Canada as an Assistant Professor in 1964. From 1968 to 1978, he was an Associate Professor there. He became a Full Professor of McGill in 1979 and served the Department of Mathematics and Statistics until he took early retirement in 2000. He is also the founder of the Canadian Journal of Statistics and the Statistical Science Association of Canada which became the Statistical Society of Canada. As of this date, A. M. Mathai is an Emeritus Professor of Mathematics and Statistics at McGill University, Canada (http://www.math.mcgill.ca/people/mathai), and Honorary Director of the Centre for Mathematical and Statistical Sciences, India. He has published over 300 research papers and more than 25 books on topics in mathematics, statistics, physics, astrophysics, and biology (selected titles Figure 1 to Figure 12). He is a Fellow of the Institute of Mathematical Statistics, National Academy of Sciences of India, Indian Society for Probability and Statistics, President of the Mathematical Society of India (2015-16), and an Elected Member of the International Statistical Institute. He was the Chairman of the Kerala State Statistical Commission (2014-2018) and President of the Society for Special Functions and their Applications (2018-). He was the principal organizer of the elements of Physics and Mathematics in the implementation of the annual workshops of the United Nations Basic Space Science Initiative (1991-2012; Figure 13), organized by ESA, NASA, and JAXA and hosted by member States of the United Nations for the benefit all Member States of the United Nations (Mathai \& Haubold, 2018a; Pyenson, Mathai, \& Haubold, 2019).

\section{Fractional Calculus: Reaction and Diffusion}

Fractional integrals, fractional derivatives and fractional differential equations were available only for real scalar variables. The most popular fractional integrals in the literature are Riemann-Liouville fractional integrals given by the following:

$$
{ }_{a} D_{x}^{-\alpha} f=\frac{1}{\Gamma(\alpha)} \int_{a}^{x}(x-t)^{\alpha-1} f(t) \mathrm{d} t, \Re(\alpha)>0,
$$

where $\mathfrak{R}(\cdot)$ denotes the real part of $(\cdot)$.

$$
{ }_{x} D_{b}^{-\alpha} f=\frac{1}{\Gamma(\alpha)} \int_{x}^{b}(t-x)^{\alpha-1} f(t) \mathrm{d} t, \Re(\alpha)>0 .
$$

Here $-\alpha$ in the exponent of $D$ indicates an integral. The $D$ with positive exponent ${ }_{a} D_{x}^{\alpha} f,{ }_{x} D_{b}^{\alpha} f$ is used to denote the corresponding fractional derivatives. Here (1.1) is called Riemann-Liouville left-sided or first kind fractional integral of order $\alpha$ and (2.2) is called Riemann-Liouville fractional integral of order $\alpha$ of the second kind or right-sided. If $a=-\infty$ and $b=\infty$ then (1.1) and (1.2) 
are called Weyl fractional integrals of order $\alpha$ and of the first kind and second kind, respectively, or the left-sided and right-sided ones. Mathai was trying to find an interpretation or connection of fractional integrals in terms of statistical densities and random variables. In Mathai (2009), an interpretation is given for Weyl fractional integrals as densities of sum (first kind) and difference (second kind) of independently distributed real positive random variables having special types of densities. Fractional integrals were also given interpretations as fractions of total integrals coming from gamma and type-1 beta random variables. Also Weyl fractional integrals were extended to real matrix-variate cases there.

\subsection{Mellin Convolutions of Products and Ratios}

Then while working on Mellin convolutions of products and ratios, Mathai found that a fusion of fractional calculus and statistical distribution theory was possible which also opened up ways of extending fractional calculus to real scalar functions of matrix argument, when the argument matrix is real or in the complex domain. Let us consider real scalar variables first. The Mellin convolution of a product of two functions $f_{1}\left(x_{1}\right)$ and $f_{2}\left(x_{2}\right)$ says the following: Consider the integral

$$
g_{2}\left(u_{2}\right)=\int_{v} \frac{1}{v} f_{1}\left(\frac{u}{v}\right) f_{2}(v) \mathrm{d} v .
$$

Then the Mellin transform of $g_{2}\left(u_{2}\right)$, with Mellin parameter $s$, is the product of the Mellin transforms of $f_{1}$ and $f_{2}$. That is

$$
M_{g_{2}}(s)=M_{f_{1}}(s) M_{f_{2}}(s) \text {, }
$$

where

$$
M_{f_{1}}(s)=\int_{0}^{\infty} x_{1}^{s-1} f_{1}\left(x_{1}\right) \mathrm{d} x_{1} \text { and } \int_{0}^{\infty} x_{2}^{s-1} f_{2}\left(x_{2}\right) \mathrm{d} x_{2}=M_{f_{2}}(s),
$$

whenever they exist. If $g_{2}\left(u_{2}\right)$ is written as

$$
g_{2}\left(u_{2}\right)=\int_{v} \frac{1}{v} f_{1}(v) f_{2}\left(\frac{u}{v}\right) \mathrm{d} v
$$

then also the formula in (1.4) holds. Thus, the Mellin convolution of a product has the two integral forms in (1.3) and (1.5). But, Mellin convolution of a ratio will have four different representations. Two of these are the following:

$$
M_{g_{1}}\left(u_{1}\right)=M_{f_{1}}(s) M_{f_{2}}(2-s)
$$

where

$$
g_{1}\left(u_{1}\right)=\int_{v} v f_{1}(u v) f_{2}(v) \mathrm{d} v
$$

and

$$
M_{g_{1}}(s)=M_{f_{1}}(2-s) M_{f_{2}}(s)
$$

where

$$
g_{1}\left(u_{1}\right)=\int_{v} \frac{v}{u_{1}^{2}} f_{1}\left(\frac{v}{u_{1}}\right) f_{2}(v) \mathrm{d} v .
$$




\subsection{Statistical Interpretations of Mellin Convolutions}

Let $x_{1}$ and $x_{2}$ be real scalar positive random variables, independently distributed, with densities $f_{1}\left(x_{1}\right)$ and $f_{2}\left(x_{2}\right)$, respectively. Let $u_{2}=x_{1} x_{2}$ and $u_{1}=\frac{x_{2}}{x_{1}}, v=x_{2}$. Then the Jacobians are $\frac{1}{v}$ and $-\frac{v}{u_{1}^{2}}$ respectively or

$$
\mathrm{d} x_{1} \wedge \mathrm{d} x_{2}=\frac{1}{v} \mathrm{~d} u_{2} \wedge \mathrm{d} v
$$

and

$$
\mathrm{d} x_{1} \wedge \mathrm{d} x_{2}=-\frac{v}{u_{1}^{2}} \mathrm{~d} u_{1} \wedge \mathrm{d} v
$$

The joint density of $x_{1}$ and $x_{2}$ is $f_{1}\left(x_{1}\right) f_{2}\left(x_{2}\right)$ due to statistical independence and then the marginal densities of $u_{2}$ and $u_{1}$, denoted by $g_{2}\left(u_{2}\right)$ and $g_{1}\left(u_{1}\right)$ are the following:

$$
g_{2}\left(u_{2}\right)=\int_{v} \frac{1}{v} f_{1}\left(\frac{u_{2}}{v}\right) f_{2}(v) \mathrm{d} v
$$

and

$$
g_{1}\left(u_{1}\right)=\int_{v} \frac{v}{u_{1}^{2}} f_{1}\left(\frac{v}{u_{1}}\right) f_{2}(v) \mathrm{d} v .
$$

In $u_{2}$, if $x_{1}$ is taken as $v$ then the roles of $f_{1}$ and $f_{2}$ change in (1.10). If $x_{1}$ in $u_{1}$ is taken as $v$ then we get (1.7) with the roles of $f_{1}$ and $f_{2}$ interchanged. Hence $\frac{x_{1}}{x_{2}}$ gives two forms and $\frac{x_{2}}{x_{1}}$ gives two forms for Mellin convolution of ratios. The Mellin convolutions in (1.4) and (1.8) can be interpreted in terms of random variables. $u_{2}=x_{1} x_{2}$ gives $E\left(u_{2}^{s-1}\right)=E\left(x_{1}^{s-1}\right) E\left(x_{2}^{s-1}\right)$ due to independence where $E(\cdot)$ denotes the expected value. That is,

$$
E\left(u_{2}^{s-1}\right)=\int_{0}^{\infty} u_{2}^{s-1} g_{2}\left(u_{2}\right) \mathrm{d} u_{2}=M_{g_{2}}(s) .
$$

Similarly

$$
E\left(x_{1}^{s-1}\right)=\int_{0}^{\infty} x_{1}^{s-1} f_{1}\left(x_{1}\right)=M_{f_{1}}(s) \text { and } E\left(x_{2}^{s-1}\right)=M_{f_{2}}(s) .
$$

This means $M_{g_{2}}(s)=M_{f_{1}}(s) M_{f_{2}}(s)$, which is (1.4) the Mellin convolution of a product. Now, consider $u_{1}=\frac{x_{2}}{x_{1}}$. Then $E\left(u_{1}^{s-1}\right)=E\left(x_{2}^{s-1}\right) E\left(x_{1}^{-s+1}\right)$ due to statistical independence. This means

$$
\begin{aligned}
& E\left(u_{1}^{s-1}\right)=\int_{0}^{\infty} u_{1}^{s-1} g_{1}\left(u_{1}\right) \mathrm{d} u_{1}=M_{g_{1}}(s), \\
& E\left(x_{2}^{s-1}\right)=\int_{0}^{\infty} x_{2}^{s-1} f_{2}\left(x_{2}\right) \mathrm{d} x_{2}=M_{f_{2}}(s)
\end{aligned}
$$

and 


$$
E\left(x_{1}^{-s+1}\right)=\int_{0}^{\infty} x_{1}^{-s+1} f_{1}\left(x_{1}\right) \mathrm{d} x_{1}=\int_{0}^{\infty} x_{1}^{(2-s)-1} f_{1}\left(x_{1}\right) \mathrm{d} x_{1}=M_{f_{1}}(2-s) .
$$

In other words, $M_{g_{1}}(s)=M_{f_{1}}(2-s) M_{f_{2}}(s)$, which is (1.8), one form of Mellin convolution of a ratio. Mellin convolutions of products and ratios make direct connection to product and ratio of real positive random variables.

\subsection{Mellin Convolutions, Statistical Densities and Fractional Integrals}

Let $f_{1}\left(x_{1}\right)$ and $f_{2}\left(x_{2}\right)$ be statistical densities as in Section 1.2. Let $x_{1}$ have a type- 1 beta density with parameters $(\gamma+1, \alpha)$ or

$$
f_{1}\left(x_{1}\right)=\frac{\Gamma(\gamma+1+\alpha)}{\Gamma(\gamma+1) \Gamma(\alpha)} x_{1}^{\gamma}\left(1-x_{1}\right)^{\alpha-1}, 0 \leq x_{1} \leq 1, \mathfrak{R}(\alpha)>0, \mathfrak{R}(\gamma)>-1
$$

and zero elsewhere. In statistical problems, the parameters are real but the integrals hold for complex parameters and hence the conditions are given for complex parameters. Let $f_{2}\left(x_{2}\right)=f\left(x_{2}\right)$ an arbitrary density. Then the Mellin convolution of a product is the following:

$$
\begin{aligned}
g_{2}\left(u_{2}\right) & =\int_{v} \frac{1}{v} f_{1}\left(\frac{u_{2}}{v}\right) f(v) \mathrm{d} v \\
& =\frac{\Gamma(\gamma+1+\alpha)}{\Gamma(\gamma+1)} \frac{1}{\Gamma(\alpha)} \int_{v} \frac{1}{v}\left(\frac{u_{2}}{v}\right)^{\gamma}\left(1-\frac{u_{2}}{v}\right)^{\alpha-1} f(v) \mathrm{d} v \\
& =\frac{\Gamma(\gamma+1+\alpha)}{\Gamma(\gamma+1)} K_{2, u_{2}, \gamma}^{-\alpha} f
\end{aligned}
$$

where

$$
K_{2, u_{2}, \gamma}^{-\alpha} f=\frac{u_{2}^{\gamma}}{\Gamma(\alpha)} \int_{v>u_{2}} v^{-\gamma-\alpha}\left(v-u_{2}\right)^{\alpha_{1}} f(v) \mathrm{d} v, \Re(\alpha)>0, \Re(\gamma)>-1
$$

is an Erdélyi-Kober fractional integral of order $\alpha$ and of the second kind with parameter $\gamma$. This is a direct connection among Erdélyi-Kober fractional integral of the second kind, Mellin convolution of a product and statistical density of product of two independently distributed real scalar positive random variables where one has a type- 1 beta density with parameters $(\gamma+1, \alpha)$ and the other has an arbitrary density.

\subsection{General Definition for Fractional Integrals}

Motivated by this observation, Mathai has given a new definition for fractional integrals of the first and second kinds of order $\alpha$. Let

$$
f_{1}\left(x_{1}\right)=\phi_{1}\left(x_{1}\right) \frac{1}{\Gamma(\alpha)}\left(1-x_{1}\right)^{\alpha-1}, 0 \leq x_{1} \leq 1, \Re(\alpha)>0
$$

and $f_{1}\left(x_{1}\right)=0$ elsewhere, and $f_{2}\left(x_{2}\right)=\phi_{2}\left(x_{2}\right) f\left(x_{2}\right)$ where $\phi_{1}$ and $\phi_{2}$ are pre-fixed functions and $f\left(x_{2}\right)$ is an arbitrary function, where $f_{1}$ and $f_{2}$ need not be statistical densities. Consider the Mellin convolution of a product. Then using the same notation as $g_{2}\left(u_{2}\right)$ there is 


$$
\begin{gathered}
g_{2}\left(u_{2}\right)=\int_{v} \frac{1}{v} f_{1}\left(\frac{u_{2}}{v}\right) f_{2}(v) \mathrm{d} v \\
=\int_{v} \frac{1}{v} \phi_{1}\left(\frac{u_{2}}{v}\right) \frac{1}{\Gamma(\alpha)}\left(1-\frac{u_{2}}{v}\right)^{\alpha-1} \phi_{2}(v) f(v) \mathrm{d} v \\
=\int_{v>u_{2}} \phi_{1}\left(\frac{u_{2}}{v}\right) \frac{v^{-\alpha}}{\Gamma(\alpha)}\left(v-u_{2}\right)^{\alpha-1} \phi_{2}(v) f(v) \mathrm{d} v \\
=\frac{1}{\Gamma(\alpha)} \int_{v>u_{2}}\left(v-u_{2}\right)^{\alpha-1} f(v) \mathrm{d} v \text { for } \phi_{1}=1, \phi_{2}\left(x_{2}\right)=x_{2}^{\alpha} .
\end{gathered}
$$

But (1.15) gives a Weyl fractional integral of the second kind of order $\alpha$. If $v$ is bounded above by a constant $b$ then (1.15) is a Riemann-Liouville fractional integral of the second kind of order $\alpha$. Thus, by specifying $\phi_{1}$ and $\phi_{2}$ it can be seen that all the various definitions of fractional integrals of order $\alpha$ of the second kind can be obtained from (1.14). Evidently when $\phi_{1}\left(x_{1}\right)=x_{1}^{\gamma}$ and $\phi_{2}\left(x_{2}\right)=1$ then one has (1.13) or Erdélyi-Kober fractional integral of order $\alpha$ of the second kind with parameter $\gamma$.

\subsection{First Kind Fractional Integrals}

Let $f_{1}\left(x_{1}\right)$ and $f_{2}\left(x_{2}\right)$ be as given above in Section 1.4. Consider (1.9), the Mellin convolution of a ratio. Then

$$
\begin{aligned}
g_{1}\left(u_{1}\right) & =\int_{v} \frac{v}{u_{1}^{2}} f_{1}\left(\frac{v}{u_{1}}\right) f_{2}(v) \mathrm{d} v \\
& =\int_{v} \frac{v}{u_{1}^{2}} \phi_{1}\left(\frac{v}{u_{1}}\right) \frac{1}{\Gamma(\alpha)}\left(1-\frac{v}{u_{1}}\right)^{\alpha-1} \phi_{2}(v) f(v) \mathrm{d} v .
\end{aligned}
$$

Let $\phi_{1}\left(x_{1}\right)=x_{1}^{\gamma-1}$ and $\phi_{2}=1$. Then (1.16) reduces to the following form:

$$
g_{1}\left(u_{1}\right)=\frac{u_{1}^{-\gamma-\alpha}}{\Gamma(\alpha)} \int_{v<u_{1}} v^{\gamma}\left(u_{1}-v\right)^{\alpha-1} f(v) \mathrm{d} v=K_{1, u_{1}, \gamma}^{-\alpha} f .
$$

Then

$$
g_{1}^{*}\left(u_{1}\right)=\frac{\Gamma(\gamma+\alpha)}{\Gamma(\gamma)} K_{1, u_{1}, \gamma}^{-\alpha} f, \mathfrak{R}(\alpha)>0, \mathfrak{R}(\gamma)>0
$$

is a statistical density, when $f$ ia a statistical density. This is an Erdélyi-Kober fractional integral operator of the first kind of order $\alpha$ and parameter $\gamma$, denoted by $K_{1, u_{1}, \gamma}^{-\alpha} f$. From (1.16), by specializing $\phi_{1}$ and $\phi_{2}$ one can get all the various definitions of fractional integrals of order $\alpha$ of the first kind in the real scalar case. This formal definition of fractional integrals as Mellin convolutions of ratio and product was introduced formally in Mathai (2013). A geometrical interpretation of fractional integrals as fractions of integral over a simplex in $n$-space is given in Mathai (2014).

\subsection{Extension of Fractional Integrals to Real Matrix-Variate Case}

Mathai (2009) introduced fractional integrals in the real matrix-variate case but they could not be given any physical interpretations. In Mathai (2013) there are 
interpretations in terms of statistical distribution problem and M-convolutions introduced in Mathai (1997) (Figure 11). A meaningful interpretation of $\mathrm{M}$-convolutions is given as the densities of product and ratio of matrix-variate random variables.

All the matrices appearing here are $p \times p$ real positive definite matrices, unless specified otherwise. The notation $X_{j}>O$ means the $p \times p$ real symmetric matrix, $X_{j}=X_{j}^{\prime}$, is positive definite. $X_{j}^{\overline{2}}$ means the positive definite square root of the positive definite matrix $X_{j}$. If $X=\left(x_{i j}\right)$ is $m \times n$ then the wedge product of differentials will be denoted by $\mathrm{d} X$, that is,

$$
\mathrm{d} X=\prod_{i=1}^{m} \prod_{j=1}^{n} \wedge \mathrm{d} x_{i j}
$$

and it is $\prod_{i \geq j=1}^{p} \wedge \mathrm{d} x_{i j}$ if $m=n=p$ and $X=X^{\prime}$. Also,

$\int_{A}^{B} f(X) \mathrm{d} X=\int_{A<X<B} f(X) \mathrm{d} X$ means the integral over all $X>O$ of the real-valued scalar function $f(X)$ of $X$, such that

$A>O, B>O, X-A>O, B-X>O$ where $A$ and $B$ are positive definite constant matrices. Here Jacobians of matrix transformations are needed. These will be given as lemmas, without proofs. For proofs and for other such Jacobians see Mathai (1997).

Lemma 1.1. Let $X=\left(x_{i j}\right)$ be $m \times n$ matrix of distinct real scalar variables $x_{i j}$ 's. Let $A$ be $m \times m$ and $B$ be $n \times n$ nonsingular constant matrices. Then

$$
Y=A X B \Rightarrow \mathrm{d} Y=|A|^{n}|B|^{m} \mathrm{~d} X
$$

where $|(\cdot)|$ denotes the determinant of $(\cdot)$.

Lemma 1.2. Let $X=X^{\prime}$ be $p \times p$. Let $A$ be a $p \times p$ nonsingular constant matrix. Then

$$
Y=A X A^{\prime} \Rightarrow \mathrm{d} Y=|A|^{p+1} \mathrm{~d} X .
$$

Lemma 1.3. Let $X$ be a $p \times p$ nonsingular matrix. Let $Y=X^{-1}$. Then

$$
Y=X^{-1} \Rightarrow \mathrm{d} Y= \begin{cases}|X|^{-2 p} \mathrm{~d} X, & \text { for a general } X \\ |X|^{-(p+1)} \mathrm{d} X, & \text { for } X=X^{\prime}\end{cases}
$$

Lemma 1.4. Let $X>O$ be $p \times p$. Let $T=\left(t_{i j}\right)$ be a lower triangular matrix with positive diagonal elements, that is,

$$
\begin{aligned}
& t_{i j}=0, i<j, t_{j j}>0, j=1, \cdots, p,-\infty<t_{i j}<\infty, i>j . \text { Then } \\
& X=T T^{\prime} \Rightarrow \mathrm{d} X=2^{p}\left\{\prod_{j=1}^{p} t_{j j}^{p+1-j}\right\} \mathrm{d} T \text {. }
\end{aligned}
$$

With the help of (1.22) one can evaluate a matrix-variate gamma integral and write the result as

$$
\Gamma_{p}(\alpha)=\pi^{\frac{p(p-1)}{4}} \Gamma(\alpha) \Gamma\left(\alpha-\frac{1}{2}\right) \cdots \Gamma\left(\alpha-\frac{p-1}{2}\right), \Re(\alpha)>\frac{p-1}{2}
$$

where the real matrix-variate gamma integral is 


$$
\int_{X>0}|X|^{\alpha-\frac{p+1}{2}} \mathrm{e}^{-\operatorname{tr}(X)} \mathrm{d} X=\Gamma_{p}(\alpha), \Re(\alpha)>\frac{p-1}{2}
$$

with $\operatorname{tr}(\cdot)$ denoting the trace of $(\cdot)$. Apply Lemma 1.4 to $X$ in the integrand of (1.24). Then the integral splits into integrals over $t_{j j}$ 's and $t_{i j}$ 's, for $i>j$, and both types of integrals can be evaluated by using a real scalar variable gamma integral. Then the final result is that of (1.23). Combining (1.24) and (1.20) one can define a matrix-variate gamma density as

$$
h_{1}(X)=\left\{\begin{array}{l}
\frac{|B|^{\alpha}}{\Gamma_{p}(\alpha)}|X|^{\alpha-\frac{p+1}{2}} \mathrm{e}^{-\mathrm{tr}(B X)} \mathrm{d} X, X>O, B>O, \Re(\alpha)>\frac{p-1}{2} \\
0, \text { elsewhere. }
\end{array}\right.
$$

Since the total integral is 1 , the identity follows from (1.25),

$$
|B|^{-\alpha} \equiv \frac{1}{\Gamma_{p}(\alpha)} \int_{X>0}|X|^{\alpha-\frac{p+1}{2}} \mathrm{e}^{-\operatorname{tr}(B X)} \mathrm{d} X, B>O, \Re(\alpha)>\frac{p-1}{2} .
$$

This identity will be used to establish fractional derivatives in a class of matrix-variate functions. The real matrix-variate type- 1 beta density is defined as

$h_{2}(X)=\left\{\begin{array}{l}\frac{\Gamma_{p}(\alpha+\beta)}{\Gamma_{p}(\alpha) \Gamma_{p}(\beta)}|X|^{\alpha-\frac{p+1}{2}}|I-X|^{\beta-\frac{p+1}{2}}, O<X<I, \Re(\alpha)>\frac{p-1}{2}, \Re(\beta)>\frac{p-1}{2} \\ 0, \text { elsewhere. }\end{array}\right.$

There is a corresponding type- 2 beta density, which is of the form

$$
h_{3}(X)=\left\{\begin{array}{l}
\frac{\Gamma_{p}(\alpha+\beta)}{\Gamma_{p}(\alpha) \Gamma_{p}(\beta)}|X|^{\alpha-\frac{p+1}{2}}|I+X|^{-(\alpha+\beta)}, X>O, \Re(\alpha)>\frac{p-1}{2}, \Re(\beta)>\frac{p-1}{2} \\
0, \text { elsewhere. }
\end{array}\right.
$$

\subsection{Fractional Integrals for the Real Matrix-Variate Case}

With the preliminaries in Section 1.6 one can define fractional integrals in the real matrix-variate case. Let $X_{1}>O$ and $X_{2}>O$ be $p \times p$ real matrix-variate random variables, independently distributed. Let $U_{2}=X_{2}^{\frac{1}{2}} X_{1} X_{2}^{\frac{1}{2}}$ be defined as the symmetric product of $X_{1}$ and $X_{2}$ and let $U_{1}=X_{2}^{\frac{1}{2}} X_{1}^{-1} X_{2}^{\frac{1}{2}}$ be defined as the symmetric ratio of $X_{2}$ over $X_{1}$. Let $V=X_{2}$. Then with the help of the above lemmas one can show that, ignoring sign,

$$
\mathrm{d} X_{1} \wedge \mathrm{d} X_{2}=|V|^{-\frac{p+1}{2}} \mathrm{~d} U_{2} \wedge \mathrm{d} V, \mathrm{~d} X_{1} \wedge \mathrm{d} X_{2}=|V|^{\frac{p+1}{2}}\left|U_{1}\right|^{-(p+1)} \mathrm{d} U_{1} \wedge \mathrm{d} V .
$$

Denoting the densities of $U_{2}$ and $U_{1}$ as $g_{2}\left(U_{2}\right)$ and $g_{1}\left(U_{1}\right)$, one can compute these by using the densities $f_{1}\left(X_{1}\right)$ and $f_{2}\left(X_{2}\right)$ through transformation of variables and they will be the following:

$$
g_{2}\left(U_{2}\right)=\int_{V}|V|^{-\frac{p+1}{2}} f_{1}\left(V^{-\frac{1}{2}} U_{2} V^{-\frac{1}{2}}\right) f_{2}(V) \mathrm{d} V
$$


and

$$
g_{1}\left(U_{1}\right)=\int_{V}|V|^{\frac{p+1}{2}}\left|U_{1}\right|^{-(p+1)} f_{1}\left(V^{\frac{1}{2}} U_{1}^{-1} V^{\frac{1}{2}}\right) f_{2}(V) \mathrm{d} V .
$$

Let $f_{1}\left(X_{1}\right)$ be a type- 1 beta density of the type in (1.27) with parameters $\left(\gamma+\frac{p+1}{2}, \alpha\right)$. Note that in (1.27) the parameters are $(\alpha, \beta)$. Then $g_{2}\left(U_{2}\right)$ will be of the following form:

$$
\begin{aligned}
g_{2}\left(U_{2}\right) & =\frac{\Gamma_{p}\left(\gamma+\frac{p+1}{2}+\alpha\right)}{\Gamma_{p}\left(\gamma+\frac{p+1}{2}\right)} \frac{\left|U_{2}\right|^{\gamma}}{\Gamma_{p}(\alpha)} \int_{V>U_{2}}|V|^{-\alpha-\gamma}\left|V-U_{2}\right|^{\alpha-\frac{p+1}{2}} f(V) \mathrm{d} V \\
& =\frac{\Gamma_{p}\left(\gamma+\frac{p+1}{2}+\alpha\right)}{\Gamma_{p}\left(\gamma+\frac{p+1}{2}\right)} K_{2, U_{2}, \gamma}^{-\alpha} f .
\end{aligned}
$$

This $K_{2, U_{2}, \gamma}^{-\alpha} f$ in (1.32) for $p=1$ is an Erdélyi-Kober fractional integral of the second kind of order $\alpha$ and parameter $\gamma$ and hence Mathai called the integral as Erdélyi-Kober fractional integral of order $\alpha$ and parameter $\gamma$ of the second kind in the real matrix-variate case. In a similar fashion, Erdélyi-Kober fractional integral of order $\alpha$ of the first kind with parameter $\gamma$, available from (1.31) by taking $f_{1}\left(X_{1}\right)$ as a real matrix-variate type-1 beta with parameters $(\gamma, \alpha)$ is the following:

$$
K_{1, U_{1}, \gamma}^{-\alpha} f=\frac{\left|U_{1}\right|^{-\gamma-\alpha}}{\Gamma_{p}(\alpha)} \int_{V<U_{1}}|V|^{\gamma}\left|U_{1}-V\right|^{\alpha-\frac{p+1}{2}} f(V) \mathrm{d} V .
$$

The density of $U_{1}$, again denoted by $g_{1}\left(U_{1}\right)$, is given by

$$
g_{1}\left(U_{1}\right)=\frac{\Gamma_{p}(\gamma+\alpha)}{\Gamma_{p}(\gamma)} K_{1, U_{1}, \gamma}^{-\alpha} f
$$

where the first kind Erdélyi-Kober fractional integral in the matrix-variate case is given in(1.33). The above notations as well as a unified notation for fractional integrals and fractional derivatives were introduced by Mathai (2013, 2014, 2015).

The above results in the real matrix-variate case are extended to complex matrix-variate cases, see Mathai (2013), to many matrix-variate cases, see Mathai (2014) and also the corresponding fractional derivatives in the matrix-variate case are worked out in Mathai (2015). The matrix differential operator introduced in Mathai (2015) is not a universal one, even though it works on some wide classes of functions. The matrix differential operator is introduced through the following symbolic representation. Let $D$ be a differential operator defined for real matrix-variate case. Then $D^{\alpha}$ and $D^{-\alpha}$ represent $\alpha$ th order fractional derivative and fractional integral respectively. Then

$$
D^{\alpha} f=D^{n} D^{-(n-\alpha)} f, n=1,2, \cdots, \mathfrak{R}(n-\alpha)>\frac{p-1}{2}, \mathfrak{R}(\alpha)>\frac{p-1}{2} .
$$


This is the $\alpha$ th order fractional derivative in Riemann-Liouville sense. Consider

$$
D^{\alpha} f=D^{-(n-\alpha)} D^{n} f, n=1,2, \cdots, \Re(\alpha)>\frac{p-1}{2}, \Re(n-\alpha)>\frac{p-1}{2}
$$

is the $\alpha$ th order fractional derivative in the Caputo sense. In the Caputo case, $D^{n}$ operates on $f$ first and then the fractional integral $D^{-(n-\alpha)}$ is taken, whereas in the Riemann-Liouville sense, the $(n-\alpha)$ th order fractional integral is taken first and then $D^{n}$ operates on this. A universal differential operator $D$ in the real as well as complex matrix-variate case is still an open problem for further research.

\section{Krätzel Integral: Thermonuclear Functions}

Let $x$ be a real scalar positive variable. Consider the integrals

$$
I_{1}=\int_{0}^{\infty} x^{\gamma-1} \mathrm{e}^{-a x^{\delta}-b x^{\rho}} \mathrm{d} x, a>0, b>0, \delta>0, \rho>0
$$

and

$$
I_{2}=\int_{0}^{\infty} x^{\gamma-1} \mathrm{e}^{-a x^{\delta}-b x^{-\rho}}, a>0, b>0, \delta>0, \rho>0 .
$$

Structures such as the ones in (2.1) and (2.2) appear in many different areas. This (2.2) for $\delta=1, \rho=1$ is the basic Krätzel integral, see Krätzel (1979). For $\rho=1$ and general $\delta>0$ is the generalized Krätzel integral. An integral transform of the form

$$
I_{3}=\int_{0}^{\infty} \mathrm{e}^{-a x-b x^{-1}} f(x) \mathrm{d} x
$$

where $f(x)$ is arbitrary so that $I_{3}$ exists, is known as Krätzel transform. Mathai has investigated various aspects of (2.1) and (2.2) in detail and he has also introduced a statistical density in terms of Krätzel integral. The structures in (2.2) and (2.1) can be generated as Melin convolutions of product and ratio. Consider the real scalar variables $x_{1}$ and $x_{2}$ and the corresponding functions $f_{1}\left(x_{1}\right)$ and $f_{2}\left(x_{2}\right)$. Then it is seen from (1.10) that the Mellin convolution of a product is given by

$$
g_{2}\left(u_{2}\right)=\int_{v} \frac{1}{v} f_{1}\left(\frac{u_{2}}{v}\right) f_{2}(v) \mathrm{d} v \text { and } M_{g_{2}}(s)=M_{f_{1}}(s) M_{f_{2}}(s)
$$

or $u_{2}=x_{1} x_{2}, v=x_{2}$, and the Mellin convolution of a ratio, from (1.7), as

$$
g_{1}\left(u_{1}\right)=\int_{v} v f_{1}\left(u_{1} v\right) f_{2}(v) \mathrm{d} v \text { and } M_{g_{1}}(s)=M_{f_{1}}(s) M_{f_{2}}(2-s)
$$

or $u_{1}=\frac{x_{1}}{x_{2}}, v=x_{2}$. Let $f_{1}$ and $f_{2}$ be generalized gamma functions of the form

$$
f_{j}\left(x_{j}\right)=x_{j}^{\alpha_{j}-1} \mathrm{e}^{-a_{j} x_{j}^{\beta_{j}}}, x_{j}>0, a_{j}>0, \beta_{j}>0, j=1,2 .
$$

Then $g_{1}\left(u_{1}\right)$ of $(2.5)$ reduces to the form

$$
g_{1}\left(u_{1}\right)=u_{1}^{\alpha_{1}-1} \int_{0}^{\infty} v^{\alpha_{1}+\alpha_{2}-1} \mathrm{e}^{-a_{1}\left(u_{1} v\right)^{\beta_{1}}-a_{2} v^{\beta_{2}}} \mathrm{~d} v .
$$


This is the form in (2.1). Now, consider Mellin convolution of a product when $f_{1}$ and $f_{2}$ are generalized gamma functions in (2.6). Then $g_{2}\left(u_{2}\right)$ reduces to the form

$$
g_{2}\left(u_{2}\right)=u_{2}^{\alpha_{1}-1} \int_{0}^{\infty} v^{\alpha_{2}-\alpha_{1}-1} e^{-a_{1}\left(\frac{u_{2}}{v}\right)^{\beta_{1}}-a_{2} v^{\beta_{2}}} \mathrm{~d} v .
$$

This is the form in (2.2). Hence (2.1) and (2.2) can be treated as Mellin convolutions of ratio and product when $f_{1}$ and $f_{2}$ are generalized gamma functions.

Note that if $f_{1}$ and $f_{2}$ are multiplied by the corresponding normalizing constants $c_{1}$ and $c_{2}$ then $f_{1}$ and $f_{2}$ become statistical densities. Let $x_{1}$ and $x_{2}$ be independently distributed real scalar positive random variables. Let $u_{2}=x_{1} x_{2}, u_{1}=\frac{x_{1}}{x_{2}}, v=x_{2}$. Then the densities of $u_{2}$ and $u_{1}$ are given by (2.4) and (2.5) multiplied by the appropriate constants and reduce to the forms in (2.8) and (2.7), multiplied by appropriate constants. In other words, (2.1) and (2.2), multiplied by appropriate constants, can be looked upon as the density of a ratio and product respectively.

The integrand in (2.2) for $\delta=1, \rho=1, \gamma=-\frac{1}{2}$ and normalized is the inverse Gaussian density available in stochastic processes. The integral in (2.2) for $\delta=1, \rho=\frac{1}{2}$ is the basic reaction-rate probability integral, which will be considered later. Mathai (2012) has introduced a Krätzel density associated with (2.1) and (2.2) and it is shown that one has general Bayesian structures in (2.1) and (2.2). For example, let us consider a conditional density of $y$, given $x$, in the form

$$
h_{1}(y \mid x)=\hat{c}_{1} y^{\alpha} \mathrm{e}^{-a\left(\frac{y}{x}\right)^{\rho}}, y>0, x>0, a>0
$$

and $\hat{c}_{1}$ can act as the normalizing constant. In other words, the conditional density is a generalized gamma density. Let the marginal density of $x$ be given by $h_{2}(x)=\hat{c}_{2} x^{\beta} \mathrm{e}^{-a_{1} x^{\delta}}, a>0, x>0, \delta>0$, and $\hat{c}_{2}$ can act as a normalizing constant, a generalized gamma density. Then the joint density of $y$ and $x$ is given by

$$
h_{1}(y \mid x) h_{2}(x)=\hat{c}_{1} \hat{c}_{2} y^{\alpha} x^{\beta} \mathrm{e}^{-a_{1} x^{\delta}-a\left(\frac{y}{x}\right)^{\rho}} .
$$

Then the unconditional density of $y, f_{y}(y)$, is available by integrating out $x$ from this joint density. That is,

$$
f_{y}(y)=\hat{c}_{1} \hat{c}_{2} y^{\alpha} \int_{0}^{\infty} x^{\beta} \mathrm{e}^{-a_{1} x^{\delta}-a \frac{y^{\delta}}{x^{\delta}}} \mathrm{d} x .
$$

Now, compare (2.2) and (2.11). They are of one and the same forms. Hence (2.2), multiplied by an appropriate constant, can be considered as an unconditional density in a Bayesian structure.

For $\delta=1, \rho=1$ in (2.1) and (2.2) one can extend the integrals to the real and complex matrix-variate cases. Mathai has also looked into this problem of Krätzel integrals in the matrix-variate cases. There will be difficulty with the Ja- 
cobians if one considers general parameters $\delta$ and $\rho$ in the matrix-variate case. The type of difficulties that can arise is described in Mathai (1997) by considering the transformation $Y=X^{2}$ when $X=X^{\prime}$. In the real matrix-variate case the scalar quantity $X^{\gamma}$ is replaced by the determinant $|X|^{\gamma}$ and exponent $\mathrm{e}^{-a x}$ is replaced by $\mathrm{e}^{-a \operatorname{tr}(X)}$ for $X>O$ or $\mathrm{e}^{-\operatorname{tr}(A X)}$ if $a$ is also replaced by a positive definite constant matrix $A>O$. Mathai has also extended Baysian structures, densities of product and ratio, inverse Gaussian density, Krätzel integral and Krätzel density, to matrix-variate cases. When the matrix is in the complex domain $|X|$ is replaced by $|\operatorname{det}(\tilde{X})|=$ absolute value of the determinant of $\tilde{X}$, where $\tilde{X}$ is a matrix in the complex domain.

\section{Pathway Model: Entropy, Probability, Dynamics}

In a physical system the stable solution may be exponential or power function or Gaussian. This is the idealized situation. But in reality the solution may be somewhere nearby the ideal or the stable situation. In order to capture the ideal situation as well as the neighboring unstable situations, a model with a switching mechanism was introduced by Mathai (2005). A form of this was proposed in the 1970's by Mathai in connection with population studies. This was a scalar variable case. Then the ideas were extended to matrix-variate cases and brought out in 2005. For the real scalar positive variable situation, the model is the following:

$$
p_{1}(x)=\tilde{c}_{1} x^{\gamma}\left[1-a(1-q) x^{\delta}\right]^{\frac{\eta}{1-q}}, a>0, \delta>0, q<1, \eta>0, x>0 .
$$

If (3.1) is to be used as a statistical density then $\tilde{c}_{1}$ is the normalizing constant there. Otherwise $\tilde{c}_{1}$ is a constant, may be $\tilde{c}_{1}=1$ and then (3.1) will be a mathematical model. For $q>1$ we can write $1-q=-(q-1)$ and then (3.1) becomes

$$
p_{2}(x)=\tilde{c}_{2} x^{\gamma}\left[1+a(q-1) x^{\delta}\right]^{-\frac{\eta}{q-1}}, a>0, x>0, q>1
$$

When $q \rightarrow 1$ then $p_{1}(x)$ and $p_{2}(x)$ go to

$$
p_{3}(x)=\tilde{c}_{3} x^{\gamma} \mathrm{e}^{-a \eta x^{\delta}}, a>0, x>0 \text {. }
$$

Note that $p_{1}(x)$ in (3.1) is in the family of generalized type-1 beta family of functions, whereas $p_{2}(x)$ is in the family of generalized type- 2 beta family of functions and $p_{3}(x)$ belongs to the generalized gamma family of functions. Thus, when the pathway parameter $q$, goes from $-\infty$ to 1 we have one family of functions, when $q$ is from 1 to $\infty$ we have another family of functions and when $q \rightarrow 1$ we have a third family of functions. Thus, all the three cases are contained in (3.1), which is the pathway model for the real positive scalar variable case. Replace $x$ by $|x|,-\infty<x<\infty$, to extend the families over the real line.

When $p_{1}(x), p_{2}(x), p_{3}(x)$ are statistical densities, then (3.1) to (3.3) give a distributional pathway. Mathai has also established a parallel pathway in terms 
of entropy optimization and in terms of differential equations. These give entropic and differential pathways as well. For example, consider the optimization of Mathai's entropy, namely

$$
M_{\alpha}(f)=\frac{\int_{x}[f(x)]^{\frac{\eta+1-q}{\eta}} \mathrm{d} x-1}{q-1}, q \neq 1, \eta>0, q<1+\eta
$$

where $f(x)$ is a density function of $x$, and $x$ can be real scalar or vector or matrix variable. A density means that $f(x) \geq 0$ for all $x$ and $\int_{x} f(x) \mathrm{d} x=1$. If one takes the limit when $q \rightarrow 1$ then (3.4), for real scalar $x$, reduces to

$$
M_{\alpha}(f) \rightarrow-\frac{1}{\eta} \int_{x} f(x) \ln f(x) \mathrm{d} x=S(f)
$$

where $S(f)$ is Shannon's entropy or measure of "uncertainty" or the complement of "information". In (3.5), $\int_{x} f(x) \ln f(x) \mathrm{d} x$ is taken as zero when $f(x)=0$. Consider the optimization of (3.4) subject to the conditions (a): $\int_{x} x^{\gamma(1-q)+\delta} f(x) \mathrm{d} x=$ fixed and $(\mathrm{b}): \int_{x} x^{\gamma(1-q)} f(x) \mathrm{d} x=$ fixed. For $\gamma=0$, condition (b) becomes $\int_{x} f(x) \mathrm{d} x=1$ since the total probability is 1 . For $\gamma=0, \delta=1$, (a) means that the first moment is fixed. This can correspond to the physical law of conservation of energy when dealing with energy distribution. If one uses calculus of variation to optimize (3.4) then the Euler equation is

$$
\frac{\partial}{\partial f}\left[f^{\frac{1+\eta-q}{\eta}}-\lambda_{1} x^{\gamma(1-q)} f+\lambda_{2} x^{\gamma(1-q)+\delta} f\right]=0
$$

where $\lambda_{1}$ and $\lambda_{2}$ are Lagrangian multipliers. Note that (3.6) gives the structure

$$
f^{\frac{1-q}{\eta}}=\mu_{1} x^{\gamma(1-q)}\left[1-\mu_{2} x^{\delta}\right]
$$

for some $\mu_{1}$ and $\mu_{2}$, which means

$$
f=\gamma_{1} x^{m}\left[1-\gamma_{2} X^{\delta}\right]^{\frac{\eta}{1-q}}
$$

for some $\gamma_{1}$ and $\gamma_{2}$. For $\gamma_{2}=a(1-q)$ and $\gamma_{1}=\tilde{c}_{1}$ one has the model in (3.1) with $\gamma \eta$ replaced by $\gamma$. Thus, for $q<1, q>1, q \rightarrow 1$ one has an entropic pathway. Similarly one can consider the corresponding differential equations to obtain a differential pathway.

The original paper Mathai (2005) deals with rectangular matrix-variate case. Let $X=\left(x_{i j}\right)$ be $m \times n, m \leq n$ and of rank $m$ be a matrix of distinct real scalar variables $\quad x_{i j}$ 's. Let $A$ be $m \times m$ and $B$ be $n \times n$ constant positive definite matrices. Consider the function

$$
P_{1}(X)=C_{1}\left|A X B X^{\prime}\right|^{\gamma}\left|I-a(1-q) A X B X^{\prime}\right|^{\frac{1}{1-q}}, q<1, a>0
$$

where $a>0, q<1$ are scalars, $I$ is a $m \times m$ identity matrix and $C_{1}$ is a constant. If (3.8) is to be taken as a density then $C_{1}$ is the normalizing constant there. For $q>1, P_{1}(X)$ goes to 


$$
P_{2}(X)=C_{2}\left|A X B X^{\prime}\right|^{\gamma}\left|I+a(q-1) A X B X^{\prime}\right|^{-\frac{1}{q-1}}, q>1, a>0
$$

and when $q \rightarrow 1, P_{1}(X)$ and $P_{2}(X)$ go to

$$
P_{3}(X)=C_{3}\left|A X B X^{\prime}\right|^{\gamma} \mathrm{e}^{-\operatorname{atr}\left(A X B X^{\prime}\right)}, a>0 .
$$

If a location parameter matrix is to be introduced then replace $X$ by $X-M$ where $M$ is a $m \times n$ constant matrix.

Note that the structure $\left|A X B X^{\prime}\right|$ is the structure of the volume content of a parallelotope in Euclidean $n$-space. Look at the $m$ rows of $X$. These are $1 \times n$ vectors. These can be taken as $m$ points in $n$-dimensional Euclidean space. These $m$ vectors, $m \leq n$, are linearly independent when the rank of $X$ is $m$. These taken in a given order can form a convex hull and a $m$-parallelotope. The volume of this m-parallelotope is the determinant $\left|X X^{\prime}\right|^{\frac{1}{2}}$. Hence $\left|A X B X^{\prime}\right|^{\frac{1}{2}}$ is the volume content of a generalized $m$-parallelotope.

Also $A X B X^{\prime}$ is a generalized quadratic form. For $A=I_{m}$ and $m=1$ it is a quadratic form in the $1 \times n$ vector variable. Thus the theory of quadratic form and generalized quadratic form can be extended to a wider class represented by the pathway model (3.8). The current theory of quadratic form and bilinear form in random variables is confined to samples coming from a Gaussian population, see Mathai and Provost (1992), Mathai, Provost and Hayakawa (1995) (Figure 10). The results on quadratic and bilinear forms can now be extended to the wider class of pathway models. One problem in this direction is discussed in Mathai (2007). The matrix-variate pathway model in Mathai (2005) is extended to complex domain in Mathai and Provost $(2005,2006)$. Some works in the scalar complex variable case, associated with normal or Gaussian population, are available in the literature with applications in sonar, radar, communication and engineering problems. Some applications of hermitian forms, corresponding to the quadratic forms in Mathai and Provost (1992), in light scattering and quantum mechanics are also available in the literature.

Note that (3.8) for $a=1, q=0$ is a matrix-variate type-1 beta density or $A X B X^{\prime}$ is a type- 1 beta matrix. This is the exact form of the matrix appearing in the generalized analysis of variance and design of experiments areas, in the likelihood ratio test involving one or more multivariate normal or Gaussian populations etc, a summary of the contributions of Mathai and his co-workers is available from Mathai and Saxena (1973). The theory available there is based on Gaussian populations. Now, generalized analysis of variance can be examined in a wider pathway family so that the limiting form corresponding to (3.10), will be the Gaussian case.

While exploring a reliability problem, Mathai (2003) came across a multivariate family of densities, which could be taken as a generalization of type-1 Dirichlet family of densities. Then Mathai and his co-workers introduced several generalizations of type- 1 and type-2 Dirichlet densities, see for example Thomas and Mathai (2009). For the different generalizations of type-1 and type-2 Di- 
richlet family, a number of characterization results are established showing that these models could also be generated by products of statistically independently distributed real scalar random variables. This is exactly the same structure available in the likelihood ratio criteria in the null cases of testing hypotheses on the parameters of one or more Gaussian populations as well as in the determinant $\left|A X B X^{\prime}\right|$ or in the model (3.8) for $a=1, q=0$. Thus, it is already shown that these three areas are connected.

In (3.1) if one puts $\gamma=0, \delta=1$ then one gets Tsallis statistics in non-extensive statistical mechanics. Also, (3.2) for $\delta=1$ as well as for some general $\delta>0$ is superstatistics (Beck, 2004; Cohen, 2004). (3.2) and its limiting form (3.3) are covered in superstatistics but (3.1) is not covered because superstatistics considerations deal with a conditional density of generalized gamma form as well as the marginal density a generalized gamma form then the unconditional density, which is superstatistics in statistical terms from a Bayesian point of view, can only produce a type-2 beta form, namely (3.2) form and not (3.1) form. Thus, superstatistics is also a special case of the pathway model in the real scalar positive variable case.

In the pathway idea itself there is an open area which is not yet explored. The scalar version of the pathway model in (3.1) to (3.3) can be looked upon as the behavior of a hypergeometric series ${ }_{1} F_{0}$ (binomial series) going to ${ }_{0} F_{0}$ (exponential series). That is,

$$
\begin{gathered}
{ }_{1} F_{0}\left(-\frac{1}{1-q} ; ; a(1-q) x^{\delta}\right)=\left[1-a(1-q) x^{\delta}\right]^{\frac{1}{1-q}} . \\
\lim _{q \rightarrow 1_{-}} F_{0}\left(-\frac{1}{1-q} ; ; a(1-q) x^{\delta}\right)=\mathrm{e}^{-a x^{\delta}}={ }_{0} F_{0}\left(; ;-a x^{\delta}\right) .
\end{gathered}
$$

From the point of view of a hypergeometric series, the process (3.11) to (3.12) is the process of a binomial series going to an exponential series. But a Bessel series ${ }_{0} F_{1}$ can also be sent to an exponential series. For example, consider the Bessel series

$$
\lim _{q \rightarrow 1-} F_{1}\left(; \frac{1}{1-q} ;-\frac{a}{1-q} x^{\delta}\right)=\mathrm{e}^{-a x^{\delta}}={ }_{0} F_{0}\left(; ;-a x^{\delta}\right) .
$$

Therefore, a generalized form, covering the path towards the exponential form $\mathrm{e}^{-a x^{\delta}}$, is also a Bessel form ${ }_{0} F_{1}\left(; \frac{1}{1-q} ;-\frac{a}{1-q} x^{\delta}\right)$. This path of a Bessel form going to an exponential form can produce a large variety of results. This area has open problems for further research.

\section{Special Functions of Matrix Argument}

A multivariate function usually means a function of many scalar variables. This is different from a matrix-variate function or a function of matrix argument. Functions of matrix argument are real-valued scalar functions $f(X)$ where $X$ is a square or rectangular matrix. For example, for a $p \times p$ matrix $X,|X|=$ 
determinant of $X, \operatorname{tr}(X)=$ trace of $X$ are real-valued scalar functions when $X$ is real. Even for a square $p \times p$ matrix $X$, the square root cannot be uniquely determined unless further conditions are imposed on $X$. If one uses the definition, $A=B B=B^{2}$ then $B=A^{\frac{1}{2}}$ the square root of $A$, one can have many candidates for $B$. For example, for a simple matrix like a $2 \times 2$ identity matrix $A=I_{2}, B_{1}, B_{2}, B_{3}, \cdots$ are square roots:

$$
A=\left[\begin{array}{ll}
1 & 0 \\
0 & 1
\end{array}\right], B_{1}=\left[\begin{array}{cc}
-1 & 0 \\
0 & 1
\end{array}\right], B_{2}=\left[\begin{array}{cc}
1 & 0 \\
0 & -1
\end{array}\right], B_{3}=\left[\begin{array}{ll}
1 & 0 \\
0 & 1
\end{array}\right] .
$$

If one restricts $A$ and $A^{\frac{1}{2}}$ to be positive definite matrices then $B_{3}$ is the only candidate here. Hence, if $X$ is $p \times p$ real positive definite or Hermitian positive definite then $X^{\frac{1}{2}}$ can be uniquely defined. Therefore, functions of matrix argument are developed mainly when the argument matrix is either real positive definite or Hermitian positive definite. There are three approaches available in the literature for functions of matrix argument, that is, real-valued scalar functions $f(X)$ of matrix argument $X$. For convenience, all the matrices appearing in this section are $p \times p$ positive definite denoted by $X>O$, real or Hermitian, unless stated otherwise. One definition is through Laplace and inverse Laplace transforms. This development is due to Herz (1955) and others. Here the basic assumption of functional commutativity is used, that is, $f(A B)=f(B A)$ even if $A B \neq B A$. For example, determinant and trace will satisfy this property. When $X$ is real symmetric then there exists an orthonormal matrix $Q$ such that $Q Q^{\prime}=I, Q^{\prime} Q=I, Q^{\prime} X Q=D=\operatorname{diag}\left(\lambda_{1}, \cdots, \lambda_{p}\right) \quad$ where $\lambda_{1}, \cdots, \lambda_{p}$ are the eigenvalues of $X$. Then

$$
f(X)=f(X I)=f\left(X Q Q^{\prime}\right)=f\left(Q^{\prime} X Q\right)=f(D)
$$

or $f(X)$, which is a function of $p(p+1) / 2$ real variables $x_{i j}$ 's, when $X=X^{\prime}$ and real, has become a function of $D$ which is of $p$ real variables $\lambda_{1}, \cdots, \lambda_{p}$, under this assumption of functional commutativity. If $X=\left(x_{i j}\right)=X^{\prime}, p \times p$ and $T=\left(t_{i j}\right)=T^{\prime}, p \times p$ then

$$
\operatorname{tr}(X T)=\sum_{j=1}^{p} x_{j j} t_{j j}+2 \sum_{i<j} x_{i j} t_{i j}
$$

Therefore

$$
\int_{X>O} \mathrm{e}^{-\operatorname{tr}(T X)} f(X) \mathrm{d} X \neq L_{f}(T)
$$

the Laplace transform of $f(X)$ because (4.3) is not consistent with the definition of multivariate Laplace transform. In (4.2) the non-diagonal terms appear twice. In the multivariate Laplace transform, the variables and the corresponding parameters must appear only once each. If one considers a modified parameter matrix $T^{*}=\left(t_{i j}^{*}\right), t_{i j}^{*}=t_{j i}^{*}$ for all $i$ and $j$, and

$$
t_{i j}^{*}=\left\{\begin{array}{l}
t_{i i}, i=j \\
\frac{1}{2} t_{i j}, i \neq j
\end{array}, T=\left(t_{i j}\right)=T^{\prime},\right.
$$


then

$$
\int_{X>0} \mathrm{e}^{-\operatorname{tr}\left(T^{*} X\right)} f(X) \mathrm{d} X=L_{f}\left(T^{*}\right)
$$

is the Laplace transform in the real symmetric positive definite matrix-variate case, where $T^{*}$ is the parameter matrix and $\mathrm{d} X$ stands for the wedge product of the $p(p+1) / 2$ differentials $\mathrm{d} x_{i j}$ 's or

$$
\mathrm{d} X=\prod_{i \geq j} \wedge \mathrm{d} x_{i j} .
$$

Under this approach, a hypergeometric function of matrix argument, denoted by

$$
{ }_{r} F_{s}\left(a_{1}, \cdots, a_{r} ; b_{1}, \cdots, b_{s} ; X\right) \text {, }
$$

where $a_{1}, \cdots, a_{r}$ and $b_{1}, \cdots, b_{s}$ are scalar parameters and $X$ is a $p \times p$ real positive definite matrix, is defined by a Laplace and inverse Laplace pair. Under this definition, explicit forms are available only for ${ }_{0} F_{0}$ and ${ }_{1} F_{0}$. Details of the definition and properties may be seen from Herz (1955) and from Mathai (1997).

The second approach is through zonal polynomials, developed by James (1961) and Constantine (1963). Here also functional commutativity is implicitly assumed, though not stated explicitly. Under this definition, a hypergeometric series is defined as follows:

$$
{ }_{r} F_{s}\left(a_{1}, \cdots, a_{r} ; b_{1}, \cdots, b_{s} ; X\right)=\sum_{k=0}^{\infty} \sum_{K} \frac{\left(a_{1}\right)_{K} \cdots\left(a_{r}\right)_{K}}{\left(b_{1}\right)_{K} \cdots\left(b_{s}\right)_{K}} \frac{C_{K}(X)}{k !}
$$

where $C_{K}(X)$ are zonal polynomials of order $k$,

$$
\begin{aligned}
K= & \left(k_{1}, \cdots, k_{p}\right), k_{1}+k_{2}+\cdots+k_{p}=k \text { and } \\
& (a)_{K}=\prod_{j=1}^{p}\left(a-\frac{j-1}{2}\right)_{k_{j}} \text { and }(b)_{k_{j}}=b(b+1) \cdots\left(b+k_{j}-1\right),(b)_{0}=0, b \neq 0 .
\end{aligned}
$$

Here $(b)_{k_{j}}$ is the Pochhammer symbol and $(a)_{K}$ is the generalized Pochhammer symbol. All terms of the series in (4.7) are explicitly available but since zonal polynomials are complicated to compute, only the first few terms up to $k=11$ are computed. Details of zonal polynomials may be found, for example from the book Mathai, Provost and Hayakawa (1995). The definition through (4.5) and its inverse Laplace form and the definition through (4.7) are not very powerful in extending results in the univariate case to the corresponding matrix-variate case. When (4.7) is used to extend univariate results to matrix-variate cases the following two basic results will be essential. These will be stated here as lemmas without proofs.

Lemma 4.1.

$$
\int_{X>O} \mathrm{e}^{-\operatorname{tr}(Z X)}|X|^{\alpha-\frac{p+1}{2}} C_{K}(X T) \mathrm{d} X=|Z|^{-\alpha} C_{K}\left(T Z^{-1}\right) \Gamma_{p}(\alpha, K)
$$

where

$$
\Gamma_{p}(\alpha, K)=\pi^{\frac{p(p-1)}{4}} \prod_{j=1}^{p} \Gamma\left(\alpha+k_{j}-\frac{j-1}{2}\right)=\Gamma_{p}(\alpha)(\alpha)_{K}
$$


with $(\alpha)_{K}$ defined in (4.8).

\section{Lemma 4.2.}

$$
\int_{O}^{I}|X|^{\alpha-\frac{p+1}{2}}|I-X|^{\beta-\frac{p+1}{2}} C_{K}(T X) \mathrm{d} X=\frac{\Gamma_{p}(\alpha, K) \Gamma_{p}(\beta)}{\Gamma_{p}(\alpha+\beta, K)} C_{K}(T) .
$$

Starting from 1970, Mathai developed functions of matrix argument through $\mathrm{M}$-transforms and M-convolutions. Under M-transform definition, a hypergeometric function ${ }_{r} F_{s}$ with $p \times p$ matrix argument $X>O$ is defined as that class of functions $f(X)$ satisfying functional commutativity and the integral equation

$$
\int_{X>0}|X|^{\rho-\frac{p+1}{2}} f(-X) \mathrm{d} X=C \Gamma_{p}(\rho) \frac{\prod_{j=1}^{r} \Gamma_{p}\left(a_{j}-\rho\right)}{\prod_{j=1}^{s} \Gamma_{p}\left(b_{j}-\rho\right)}, \Re(\rho)>\frac{p-1}{2}
$$

where

$$
C=\frac{\prod_{j=1}^{s} \Gamma_{p}\left(b_{j}\right)}{\prod_{j=1}^{r} \Gamma_{p}\left(a_{j}\right)} .
$$

For example

$$
\int_{X>0}|X|^{\rho-\frac{p+1}{2}} f(-X) \mathrm{d} X=\Gamma_{p}(\rho), \Re(\rho)>\frac{p-1}{2} \Rightarrow f(-X)=\mathrm{e}^{-\operatorname{tr}(X)} .
$$

Since the left side in (4.12) is a function of only one parameter $\rho$, one cannot normally recover $f(X)=f(D)$ a function of $p$ scalar variables. It is conjectured that when $f(X)$ is analytic in the cone of positive definite matrices $X>O$, one has $f(X)$ uniquely recovered from the right side of (4.12). This is not established yet and also an explicit form of an inverse or $f(X)$ through the right-side of (4.12) is an open problem. But (4.12) is the most convenient form to extend univariate results on hypergeometric functions to the corresponding class of matrix-variate cases. In general, when one goes from a univariate case, such as a univariate function $\mathrm{e}^{-x}$, to a multivariate case, there is nothing called a unique multivariate analogue. Whatever be the properties of the univariate function that one wishes to preserve in the multivariate analogue, one may be able to come up with different functions as multivariate analogues of a univariate function. Hence, a class of multivariate analogues is more appropriate than a single multivariate analogue. Properties of M-transforms and properties of hypergeometric family coming from (4.12) are available in the book Mathai (1997). When Mathai introduced M-convolutions and M-transforms, details in Mathai (1997), no physical meaning could be found. Now, a physical interpretation is available for $\mathrm{M}$-convolutions as densities of products and ratios of matrix random variables, as illustrated in Sections 1.7.

\section{Geometric Probabilities: Probability Density Function}

The work until 1999 is summarized in Mathai (1999a). The work started as an off-shoot of the work in multivariate statistical analysis. Mathai noted that the moment structure for many types of random geometric configurations was that 
of product of independently distributed type- 1 beta, type-2 beta or gamma random variables. Such structures were already handled by Mathai and his co-workers in connection with problems in multivariate statistical analysis. Earlier contributions of Mathai in this area are available from Chapter 4 of the book Mathai (1999a). Then Ruben, a colleague of Mathai at McGill University, one day gave a copy of his paper showing a conjecture in geometrical probabilities, called Miles' conjecture about a re-scaled, relocated random volume, generated by uniformly distributed random points in $n$-space, as asymptotically normal when $n \rightarrow \infty$. The proof was very roundabout. Mathai noted that it could be proved easily with the help of the asymptotic expansions of gamma functions. This paper was published in Mathai (1982). Then Mathai formulated and proved parallel conjectures regarding type- 1 beta distributed, type- 2 beta distributed points and gamma distributed random points and published a series of papers. Then Mathai noted that many European researchers were working on distances between random points, and random areas when the random points are in particular shapes such as triangles, parallelograms, squares, rhombuses etc. As generalizations of all these classes of problems, Mathai generalized Buffon's clean-tile problem, the starting point of geometrical probabilities. He considered placing a ball at random in a pyramid with polygonal base, defining "at random" in terms of kinematic measure, Mathai (1999c). When mixing geometry with probability or measure theory, or in the area of stochastic geometry, the basic axioms of probability are not sufficient, as pointed out by Bertrand's or Russell's paradoxes. We need an additional axiom of invariance under Euclidean motion. Another contribution of Mathai in this area is Mathai (1999b) where he has shown that the usual complicated procedures coming from integral geometry and differential geometry are not necessary for handling certain types of random volumes but only the simple properties of functions of matrix argument and Jacobians of matrix transformation are sufficient. The procedure is illustrated in the distribution of volume content of parallelotope generated by random points in Euclidean $n$-space. The work on geometrical probabilities is currently progressing in the areas of random sets, image processing etc. The book, Mathai (1999a), only deals with distributional aspects of random geometric configurations.

As an application of geometrical probabilities, Mathai and his co-authors looked into a geography problem of city designs of rectangular grid cities, as in North America, versus circular cities as in Europe, with reference to travel distance, and the associated expense and loss of time, from suburbs to city core, see Mathai (1998), Mathai and Moschopoulos (1999a).

\section{Astrophysics: Solar Neutrinos}

After publishing the books Mathai and Saxena $(1973,1978)$ physicists were using results in special functions in their physics problems. HJH came to Montreal, Canada in 1982 with open problems on reaction-rate theory, solar models, solar 
neutrinos, and gravitational instability. The idea was to get exact analytical results and analytical models where computations and computer models were available. Mathai figured out that the problems connected with reaction-rate theory and solar neutrinos could be tackled once the following integral was evaluated explicitly (Critchfield, 1972; Fowler, 1984):

$$
I=\int_{0}^{\infty} x^{\gamma-1} \mathrm{e}^{-a x-b x^{-\frac{1}{2}}} \mathrm{~d} x, a>0, b>0 .
$$

The corresponding general integral is

$$
I(\gamma, a, b, \delta, \rho)=\int_{0}^{\infty} x^{\gamma-1} \mathrm{e}^{-a x^{\delta}-b x^{-\rho}} \mathrm{d} x, a>0, b>0, \delta>0, \rho>0 .
$$

In 1982 Mathai could not find any mathematical technique of handling (6.2) or its particular case (6.1). He noted that (6.2) could be written as a product of two integrable functions and thereby as statistical densities by multiplying with appropriate normalizing constants. Then the structure in (6.2) could be converted into the form

$$
I(\gamma, a, b, \delta, \rho)=\int_{0}^{\infty} \frac{1}{v} f_{1}\left(\frac{u}{v}\right) f_{2}(v) \mathrm{d} v
$$

and the right side of (6.3) is the density of a product of two real scalar positive independently distributed random variables with densities $f_{1}\left(x_{1}\right)$ and $f_{2}\left(x_{2}\right)$ respectively with $u=x_{1} x_{2}, v=x_{2}$. Take

$$
f_{1}\left(x_{1}\right)=c_{1} \mathrm{e}^{-x_{1}^{\rho}}, f_{2}\left(x_{2}\right)=c_{2} x_{2}^{\gamma} \mathrm{e}^{-a x_{2}^{\delta}}, x>0, a>0, \delta>0, \rho>0
$$

where $c_{1}, c_{2}$ are normalizing constants. When $u=x_{1} x_{2}$ the density of $u$, denoted by $g(u)$, is given by

$$
g(u)=c_{1} c_{2} \int_{0}^{\infty} \frac{1}{v} v^{\gamma} \mathrm{e}^{-a v^{\delta}-b v^{-\rho}} \mathrm{d} v, b=u^{\rho} .
$$

Now, it is only a matter of evaluating the density $g(u)$ by using some other method. Note that $u=x_{1} x_{2}$ where $x_{1}$ and $x_{2}$ are independently distributed means

$$
E\left(u^{s-1}\right)=E\left(x_{1}^{s-1}\right) E\left(x_{2}^{s-1}\right)
$$

where $E(\cdot)$ denotes the expected value of $(\cdot)$. Then for (6.4)

$$
E\left(x_{1}^{s-1}\right)=c_{1} \int_{0}^{\infty} x_{1}^{s-1} \mathrm{e}^{-x_{1}^{\rho}} \mathrm{d} x_{1}=c_{1} \frac{1}{\rho} \Gamma\left(\frac{s}{\rho}\right), \Re(s)>0
$$

which also shows that $c_{1}=\frac{\rho}{\Gamma\left(\frac{1}{\rho}\right)}$ since $E\left(x_{1}^{s-1}\right)$ at $s=1$ is 1 . Evaluations of $c_{1}$ and $c_{2}$ are not necessary for our procedure to hold.

$$
E\left(x_{2}^{s-1}\right)=c_{2} \int_{0}^{\infty} x_{2}^{\gamma+s-1} \mathrm{e}^{-a x_{2}^{\delta}} \mathrm{d} x_{2}=\frac{c_{2}}{\delta} \Gamma\left(\frac{\gamma+s}{\delta}\right) a^{-\left(\frac{\gamma+s}{\delta}\right)}, \mathfrak{R}(\gamma+s)>0 .
$$

Therefore 


$$
E\left(u^{s-1}\right)=\frac{c_{1} c_{2}}{\delta \rho} a^{-\frac{\gamma}{\delta}} \Gamma\left(\frac{s}{\rho}\right) \Gamma\left(\frac{\gamma+s}{\delta}\right) a^{-\frac{s}{\delta}}
$$

Hence $g(u)$ is available from the inverse Mellin transform. That is,

$$
g(u)=\frac{c_{1} c_{2}}{\delta \rho} a^{-\frac{\gamma}{\delta}} \frac{1}{2 \pi i} \int_{c-i \infty}^{c+i \infty} \Gamma\left(\frac{s}{\rho}\right) \Gamma\left(\frac{\gamma+s}{\delta}\right)\left(a^{\frac{1}{\delta}} u\right)^{-s} \mathrm{~d} s, c>0, i=\sqrt{-1} .
$$

Comparing (6.4) with (6.5) the required integral is given by the following:

$$
I(\gamma, a, b, \delta, \rho)=\int_{0}^{\infty} \frac{1}{v} v^{\gamma} \mathrm{e}^{-a v^{\delta}-b v^{-\rho}} \mathrm{d} v=\frac{1}{\delta \rho a^{\frac{\gamma}{\delta}}} H_{0,2}^{2,0}\left[\left.a^{\frac{1}{\delta}} b^{\frac{1}{\rho}}\right|_{\left(0, \frac{1}{\rho}\right),\left(\frac{\gamma}{\delta}, \frac{1}{\delta}\right)}\right] .
$$

The right side of (6.6) is a $\mathrm{H}$-function.

For the reaction-rate probability integral, $\delta=1$ and $\rho=\frac{1}{2}$. In this case, the $\mathrm{H}$-function in (6.6) reduces to a G-function and explicit computable series forms are also given by Mathai and his co-workers. Problems considered were resonant reactions, non-resonant reactions, depleted case, and high energy tail cut off. A summary of the work until 1988 is available in the research monograph Mathai and Haubold (1988). After publishing papers in physics by using statistical techniques it was realized that the density of a product of independently distributed real positive random variables was nothing but the Mellin convolution of a product. Hence, one could have applied Mellin and inverse Mellin transform techniques there. The work in this area of reaction-rate also resulted in two encyclopedia articles, see Haubold and Mathai $(1997,1998)$.

\section{Analytic Solar Models}

Another attempt was to replace the current computer model for the Sun with analytic models. The idea was to assume a basic model for the matter density distribution in the Sun or in main sequence stars which could be treated as a sphere in hydrostatic equilibrium. Let $r$ be an arbitrary distance from the center of the Sun and let $R_{\odot}$ be the radius of the Sun. Let $x=\frac{r}{R_{\odot}}$ so that $0 \leq x \leq 1$. The model for the matter density distribution is taken as

$$
f(x)=c\left(1-x^{\delta}\right)^{\gamma}
$$

where $c$ is the central core density when $x=0$. The parameters $\delta$ and $\gamma$ are selected to agree with observational data. Then by using (6.7), expressions for mass, pressure, temperature and luminosity are computed by using physical laws. Then by using known observations, or comparing with known data on mass, pressure etc the best values for $\delta$ and $\gamma$ are estimated so that close agreement is there with observational values of mass, pressure etc. Some of the results until 1988 are given in the monograph Mathai and Haubold (1988).

Another area that was looked into was the gravitational instability problem concerning the evolution of large scale structure in the Universe. The problem 
was formulated in the form of differential equations. Mathai tried to change the operator $D=\frac{\mathrm{d}}{\mathrm{d} x}$ to $x \frac{\mathrm{d}}{\mathrm{d} x}$. Then the differential equation got simplified. Then he changed the dependent variable and found that the differential equation became a particular case of G-function differential equation. This resulted in the first paper of Mathai in integer order differential equations and it was published in Mathai (1989). Then the results were applied to gravitational instability problem (Haubold \& Mathai, 1988).

Another area looked into was the solar neutrino problem (Davis Jr., 2003; Sakurai, 2014). HJH and Mathai tried to come up with appropriate models to model the solar neutrino data. Mathai had noted that the graph of the time series data looked similar to the pattern that he had seen when working on modeling of the chemical called Melatonin in human body. Usually what is observed is the residual part of what is produced minus what is consumed or converted or lost. Hence the basic model should be an input-output type model. The necessary theory is available in Mathai (1993c). The simplest input-output model is an exponential type input $x_{1}$ and an exponential type output $x_{2}$ so that the residual part $u=x_{1}-x_{2}$. When $x_{1}$ and $x_{2}$ are independently and identically exponentially distributed then $u$ has a Laplace distribution. One model HJH and Mathai tried was Laplace type random variables over time so that the graph will look like blips at equal or random points on a horizontal line. If the time-lag is shortened then the blips will start joining together. If exponential models of different intensities, that is, in the input-output model $f\left(x_{j}\right)=\theta \mathrm{e}^{-\theta x_{j}}, \theta>0, x_{j}>0, j=1,2$, if $\theta$ is different for different blips then the pattern can be brought to the pattern seen in nature or the pattern seen from the data.

\section{Special Functions of Mathematical Physics}

Mathai and his co-workers are credited with popularizing special functions, especially $\mathrm{G}$ and $\mathrm{H}$-functions, in statistics and physics. Major part of the special function work was done with co-worker Saxena. They thought that they were the first one to use $\mathrm{G}$ and $\mathrm{H}$-functions in statistical literature. But D. G. Kabe pointed out that he had expressed a statistical density in terms of a G-function in 1958. This may be the first paper in statistics where G or H-function was used. Most probably the use of $\mathrm{G}$ and $\mathrm{H}$-function in physics an engineering areas started after the publication of the books Mathai and Saxena $(1973,1978)$. The first work on the fusion of statistical distribution theory and special functions started by creating statistical densities by using generalized special functions. In this connection the most general such density is based on a product of two H-functions, which appeared in Mathai and Saxena (1969). Another area that was looked into was Bayesian structures. The unconditional density in Bayesian analysis is of the form

$$
f(x)=\int_{y} f_{1}(x \mid y) g(y) \mathrm{d} y .
$$


What are the general families of functions for $f_{1}(x \mid y)$ and $g(y)$ so that the integral in (7.1) can be evaluated? One can construct some general mixing families of $f_{1}(x \mid y)$ and $g(y)$.

Another family of problems that was looked into were the null and non-null distributions of the likelihood ratio criterion or $\lambda$-criterion for testing hypotheses on the parameters of one or more multinormal populations. Consider the $p \times 1$ vector $X_{j}$ having the density

$$
f\left(X_{j}\right)=\frac{1}{(2 \pi)^{\frac{p}{2}}|V|^{\frac{1}{2}}} \mathrm{e}^{-\frac{1}{2}\left(X_{j}-\mu\right)^{\prime} V^{-1}\left(X_{j}-\mu\right)}, V>O
$$

where $\mu$ is a constant vector, known as the mean-value vector here. For $j=1,2, \cdots, N$ if $X_{j}$ 's are independently distributed with the same density in (7.2) then we say that we have a simple random sample of size $N$ from the $p$-variate normal or Gaussian population (7.2). Suppose that we want to test a hypothesis $H_{0}: V=$ is diagonal. This is called the test for independence in the Gaussian case. Then the $\lambda$-criterion can be shown to have the structure:

$$
u=\lambda^{\frac{2}{N}}=\frac{\left|S_{1}\right|}{\left|S_{1}+S_{2}\right|}=u_{1} u_{2} \cdots u_{p}
$$

where $S_{1}$ and $S_{2}$ are independently distributed matrix-variate gamma variables of (1.25) with the same $B$. Then the structure in (7.3) is distributed as a product of independently distributed type- 1 beta random variables, $u_{1}, \cdots, u_{p}$. Then the density of $u$ can be written as a G-function of the type $G_{p, p}^{p, 0}(u)$. The density of $\lambda$ will go in terms of a $\mathrm{H}$-function. The $\mathrm{H}$-function is more or less the most generalized special function in real scalar variable case and it is defined by the following Mellin-Barnes integral and the following standard notation is used:

$$
\begin{gathered}
H_{r, s}^{m, n}(z)=H_{r, s}^{m, n}\left[\left.z\right|_{\left.\left(b_{1}, \beta_{1}\right), \cdots, b_{s}, b_{s}\right)} ^{\left(a_{1}, b_{1}\right), \ldots\left(a_{r}, \alpha_{r}\right)}\right] \\
=\frac{1}{2 \pi i} \int_{L} \phi(\rho) z^{-\rho} \mathrm{d} \rho, \sqrt{-1}
\end{gathered}
$$

where

$$
\phi(\rho)=\frac{\left\{\prod_{j=1}^{m} \Gamma\left(b_{j}+\beta_{j} \rho\right)\right\}\left\{\prod_{j=1}^{n} \Gamma\left(1-a_{j}-\alpha_{j} \rho\right)\right\}}{\left\{\prod_{j=m+1}^{s} \Gamma\left(1-b_{j}-\beta_{j} \rho\right)\right\}\left\{\prod_{j=n+1}^{r} \Gamma\left(a_{j}+\alpha_{j} \rho\right)\right\}}
$$

where $\alpha_{j}, j=1, \cdots, r ; \beta_{j}, j=1, \cdots, s$ are real and positive numbers, $a_{j}$ 's and $b_{j}$ 's are complex numbers. $L$ is a contour separating the poles of $\Gamma\left(b_{j}+\beta_{j} \rho\right), j=1, \cdots, m$ to one side and those of $\Gamma\left(1-a_{j}-\alpha_{j} \rho\right), j=1, \cdots, n$ to the other side. Existence of the contours and convergence conditions are available from the books Mathai (1993a), Mathai and Saxena (1973, 1978), Mathai and Haubold (2008), Mathai, Saxena and Haubold (2010). When $\alpha_{1}=1=\cdots=\alpha_{r}, \beta_{1}=1=\cdots=\beta_{s}$ then the $\mathrm{H}$-function reduces to a $\mathrm{G}$-function denoted as 


$$
G_{r, s}^{m, n}(z)=G_{r, s}^{m, n}\left[\left.z\right|_{b_{1}, \cdots, b_{s}} ^{a_{1}, \cdots, a_{r}}\right] .
$$

Explicit computable series forms of $G_{0, p}^{p, 0}(x), G_{p, p}^{p, 0}(x), G_{p, p}^{p, p}(x)$ and for the general $G_{r, s}^{m, n}(x)$, were given by Mathai in a series of papers. The first three forms correspond to product of independently distributed gamma variables, type- 1 beta variables and type- 2 beta variables respectively. The details of the computable representations are available in the book Mathai (1993a). This is achieved by developing an operator which can handle poles of all orders. This operator may be seen from Mathai \& Rathie (1972) and its use from Mathai (1993a). This is a modification of a procedure developed in Mathai and Rathie (1971) to handle generalized partial fractions. Let

$$
\frac{1}{\left(x-a_{1}\right)^{m_{1}}\left(x-a_{2}\right)^{m_{2}} \cdots\left(x-a_{k}\right)^{m_{k}}}=\sum_{i=1}^{k} \sum_{j=1}^{m_{i}} \frac{c_{i j}}{\left(x-a_{i}\right)^{j}}
$$

where $m_{i}=1,2, \cdots$ for $i=1,2, \cdots, k$ and the coefficients $c_{i j}$ 's are to be evaluated. The technique developed in Mathai and Rathie (1971) enables one to compute $c_{i j}$ 's explicitly.

The $\mathrm{G}$ and $\mathrm{H}$-functions are also established by Mathai for the real matrix-variate cases through $\mathrm{M}$-transforms, along with extensions of all special functions of scalar variables to the matrix-variate cases. Also Mathai extended multivariate functions such as Apple functions, Lauricella functions, Kampé de Fériet functions etc to many matrix-variate cases. Some details may be seen from Mathai (1993a, 1997).

By making use of the explicit series forms, MAPLE and MATHEMATICA have produced computer programs for numerical computations of G-functions and MATHEMATICA has a computer program for the evaluation of $\mathrm{H}$-function also. Solutions of fractional differential equations usually end up in terms of Mittag-Leffler function, its generalization as Wright's function and its generalization as $\mathrm{H}$-function. In connection with fractional differential equations for reaction, diffusion, reaction-diffusion problems $\mathrm{HJH}$, Mathai and Saxena have given solutions for a large number of situations, which may be seen from the joint works of Haubold, Mathai and Saxena (2011), see also Mathai and Haubold (2018c). In all these solutions, either Mittag-Leffler function or Wright's function or $\mathrm{H}$-function appears. Also many other physicists, mathematicians and engineers have tried other fractional partial differential equations where also the solutions are available in terms of $\mathrm{H}$-functions.

\section{A Pseudo Dirichlet Integral}

A type-1 Dirichlet integral is over a simplex

$$
\begin{gathered}
\Omega=\left\{\left(x_{1}, \cdots, x_{k}\right) \mid 0 \leq x_{j} \leq 1,0 \leq x_{1}+\cdots+x_{k} \leq 1, j=1, \cdots, k\right\}, \text { namely } \\
\int_{\Omega} x_{1}^{\alpha_{1}-1} \cdots x_{k}^{\alpha_{k}-1}\left(1-x_{1}-\cdots-x_{k}\right)^{\alpha_{k+1}-1} \mathrm{~d} x_{1} \wedge \cdots \wedge \mathrm{d} x_{k}=D_{k+1}, \\
D_{k+1}=\frac{\prod_{j=1}^{k+1} \Gamma\left(\alpha_{j}\right)}{\Gamma\left(\alpha_{1}+\cdots+\alpha_{k+1}\right)}, \Re\left(\alpha_{j}\right)>0, j=1, \cdots, k+1 .
\end{gathered}
$$


But one can construct a multivariate integral over a hypercube giving rise to the same $D_{k}$ where the integrand is different from type-1 Dirichlet format of (7.9). Mathai (2018) constructed such a function which he called it the pseudo Dirichlet function. Consider the following integral:

$$
\begin{aligned}
D_{k}= & \int_{0}^{1} \cdots \int_{0}^{1}\left(1-x_{1}\right)^{\alpha_{1}-1} \cdots\left(1-x_{k}\right)^{\alpha_{k}-1} x_{2}^{\alpha_{1}} \cdots x_{k}^{\alpha_{1}+\cdots+\alpha_{k-1}} \\
& \times\left(1-x_{1} x_{2} \cdots x_{k}\right)^{-\left(\alpha_{1}+\cdots+\alpha_{k}-1\right)} \mathrm{d} x_{1} \wedge \cdots \wedge \mathrm{d} x_{k}, \\
D_{k}= & \frac{\prod_{j=1}^{k} \Gamma\left(\alpha_{j}\right)}{\Gamma\left(\alpha_{1}+\cdots+\alpha_{k}\right)} .
\end{aligned}
$$

The method of proving this result is to expand $\left(1-x_{1} \cdots x_{k}\right)^{-\left(\alpha_{1}+\cdots+\alpha_{k}-1\right)}$ by using a binomial expansion, integrate out variables one by one and then use the properties of Gauss' hypergeometric function of argument 1 to obtain the result in (7.10). Mathai also extended the integral (7.10) to the real matrix-variate case. In the real matrix-variate case the corresponding integral gives a constant multiple of the form $C D_{k}^{*}$ where

$$
D_{k}^{*}=\frac{\prod_{j=1}^{k} \Gamma_{p}\left(\alpha_{j}\right)}{\Gamma_{p}\left(\alpha_{1}+\cdots+\alpha_{k}\right)}, \mathfrak{R}\left(\alpha_{j}\right)>\frac{p-1}{2}, C=\frac{\left[\Gamma_{p}\left(\frac{p+1}{2}\right)\right]^{2}}{\Gamma_{p}(p+1)}
$$

where $\Gamma_{p}(\alpha)$ is the real matrix-variate gamma given by the following:

$$
\Gamma_{p}(\alpha)=\pi^{\frac{p(p-1)}{4}} \Gamma(\alpha) \Gamma\left(\alpha-\frac{1}{2}\right) \cdots \Gamma\left(\alpha-\frac{p-1}{2}\right), \Re(\alpha)>\frac{p-1}{2} .
$$

The integral is the following:

$$
\begin{aligned}
C D_{k}^{*}= & \int_{O<X_{1}<I} \cdots \int_{O<X_{K}<I}\left|I-X_{1}\right|^{\alpha_{1}-\frac{p+1}{2}} \cdots\left|I-X_{k}\right|^{\alpha_{k}-\frac{p+1}{2}} \\
& \times\left|X_{2}\right|^{\alpha_{1}}\left|X_{3}\right|^{\alpha_{1}+\alpha_{2}} \cdots\left|X_{k}\right|^{\alpha_{1}+\cdots+\alpha_{k-1}}|I-A|^{-\left(\alpha_{1}+\cdots+\alpha_{k}-\frac{p+1}{2}\right)}
\end{aligned}
$$

where $A$ is a symmetric product of matrices given by

$$
A=X_{1}^{\frac{1}{2}} X_{2}^{\frac{1}{2}} \cdots X_{k-1}^{\frac{1}{2}} X_{k} X_{k-1}^{\frac{1}{2}} \cdots X_{2}^{\frac{1}{2}} X_{1}^{\frac{1}{2}}
$$

with $X_{j}^{\frac{1}{2}}$ denoting the positive definite square root of the $p \times p$ real positive definite matrix $X_{j}, j=1, \cdots, k$. The structure in (7.10) gives the same gamma product in (7.9) with $k+1$ replaced by $k$.

\section{Multivariate Statistical Analysis and Statistical Distribution Theory: Fractional Reaction and Diffusion}

In the area of multivariate analysis, almost all exact null distributions in the most general cases and a large number of non-null distributions of $\lambda$-criteria for testing hypotheses on one or more multivariate Gaussian populations and exponential populations were given by Mathai and his co-workers. The $\lambda$-criterion is explained in (7.3). Null distributions mean the distributions when the null hypotheses are assumed to hold and non-null distributions mean without the re- 
strictions imposed by the hypotheses. In the non-null situations some of the cases are still open problems. In the null cases, $u$, a one-to-one function of the $\lambda$-criterion, has usually the following representations:

$$
\begin{aligned}
& u=u_{1} \cdots u_{p} ; \\
& u=v_{1} \cdots v_{p} ; \\
& u=w_{1} \cdots w_{p}
\end{aligned}
$$

where $u_{1}, \cdots, u_{p}$ are independently distributed real scalar type-1 beta random variables, $v_{1}, \cdots, v_{p}$ are the same type of type-2 beta random variables and $w_{1}, \cdots, w_{p}$ are the same type of gamma random variables. The density of $u$ in (8.1) can be written in terms of a $G_{p, p}^{p, 0}(u)$, that of (8.2) as a $G_{p, p}^{p, p}(u)$ and that of (8.3) as a $G_{0, p}^{p, 0}(u)$. Computational aspects of these forms are already discussed in Section 7 above. In geometrical probabilities also the squares of the volume content of a p-parallelotope can be written as (8.1) when the random points are type- 1 beta distributed, as (8.2) when the random points are type- 2 beta distributed and as (8.3) when the random points are gamma distributed. There also densities can be evaluated in terms of the three types of G-functions, as explained above.

Also, Mathai and his co-workers have established a connection between $\lambda$ -criterion in testing of statistical hypotheses, connected with multivariate normal populations, and certain generalizations of type-1 Dirichlet models. Various generalizations of type- 1 and type-2 Dirichlet models were introduced by Mathai and his co-workers starting with Mathai (2003). In this area also G and $\mathrm{H}$-functions appear. The forms $G_{p, p}^{p, 0}(x)$ and $G_{p, p}^{p, p}(x)$, coming from products of scalar variables of type- 1 and type- 2 beta, appear in this area of generalized Dirichlet models.

Exact 11-digit accurate percentage points connected with the null distributions of the $\lambda$-criteria were developed by Mathai and Katiyar starting with the Biometrika paper Mathai and Katiyar (1979). As a byproduct, an algorithm for non-linear least squares was also developed by them, see Mathai and Katiyar (1993a). Mathai has contributions in integer programming and optimization also, see Kounias and Mathai (1988).

\section{Mittag-Leffler Function and Mittag-Leffler Density}

$\mathrm{HJH}$, Mathai and Saxena have solved fractional differential equations, starting from 2000, where the solutions invariably come in terms of Mittag-Leffler function, Wright's function or H-function. Exponential type solutions of integer order differential equations automatically change to Mittag-Leffler functions when we go from integer order to fractional order differential equations. There is also a Mittag-Leffler stochastic process based on a Mittag-Leffler density, which is a non-Gaussian stochastic process. Work in this area is summarized in Haubold, Mathai and Saxena (2011). Mathai has also introduced a generalized Mit- 
tag-Leffler density and has shown that it is attracted to heavy-tailed models such as Lévy and Linnik densities, rather than to Gaussian models.

\section{Characterization Problems: Gauss and Beyond}

In this area, two basic books are Mathai and Rathie (1975) and Mathai and Pederzoli (1977). Characterization is the unique determination through some given properties. Characterization of a density means to show that certain property or properties uniquely determine that density. Unique determination of a concept means to give an axiomatic definition to that concept. That is, to show that the proposed axioms will uniquely determine the concept. The techniques used in this area, to go from the given properties to the density or from the given axioms to the concept such as "uncertainty" or its complement "information" etc, are functional equations, differential equations, Laplace, Mellin, and Fourier transforms. For example, look at the distribution of error. The error $\epsilon$ may be the error in measurement in an experiment, the error between observed and predicted values etc. If the factors contributing to the error are known then the experimenter will try to control these factors. Very often the error is contributed by infinitely many unknown factors each factor contributing infinitesimal quantities towards $\epsilon$. Put some conditions on this $\epsilon$. Let

$$
\epsilon=\epsilon_{1}+\epsilon_{2}+\cdots, S_{n}=\epsilon_{1}+\cdots+\epsilon_{n}
$$

where $\epsilon_{1}, \epsilon_{2}, \cdots$ are assumed to be independently distributed. Assume that each $\epsilon_{j}=+a$ or $-a$ with equal probabilities. That is,

$$
\operatorname{Pr}\left\{\epsilon_{j}=a\right\}=\operatorname{Pr}\left\{\epsilon_{j}=-a\right\}=\frac{1}{2}, j=1,2, \cdots
$$

Assume that the total variance of $\epsilon$ is finite or

$$
\operatorname{Var}(\epsilon)=\sigma^{2}<\infty \text {. }
$$

Check the consequence of these three assumptions.

$\operatorname{Var}\left(S_{n}\right)=n \operatorname{Var}\left(\epsilon_{j}\right)=n\left[\frac{1}{2} a^{2}+\frac{1}{2}(-a)^{2}\right]=n a^{2}$, where $a$ is fixed and finite. For large $n$ one may take $a=\frac{\sigma^{2}}{n}$. The moment generating function of $\epsilon_{j}$ is

$$
M_{\epsilon_{j}}(t)=E\left[\mathrm{e}^{\epsilon_{j} t}\right]=\frac{1}{2}\left[\mathrm{e}^{-a t}+\mathrm{e}^{a t}\right]=1+\frac{a^{2} t^{2}}{2 !}+\frac{a^{4} t^{4}}{4 !}+\cdots=1+\frac{\sigma^{2} t^{2}}{2 ! n}+O\left(\frac{1}{n^{2}}\right) .
$$

Hence

$$
M_{S_{n}}(t)=\left[M_{\epsilon_{j}}(t)\right]^{n}=\left[1+\frac{\sigma^{2} t^{2}}{2 ! n}+O\left(\frac{1}{n^{2}}\right)\right]^{n} .
$$

That is

$$
\ln \left[M_{S_{n}}(t)\right]=n \ln \left[1+\frac{\sigma^{2} t^{2}}{2 ! n}+O\left(\frac{1}{n^{2}}\right)\right]=\frac{\sigma^{2} t^{2}}{2}+O\left(\frac{1}{n}\right) \rightarrow \frac{\sigma^{2} t^{2}}{2} \text { as } n \rightarrow \infty .
$$

Therefore, as $n \rightarrow \infty$, 


$$
M_{\epsilon}(t)=\mathrm{e}^{\frac{t^{2} \sigma^{2}}{2}}
$$

which is the moment generating function of a normal density with mean value zero and variance $\sigma^{2}$ or the density is

$$
f(\epsilon)=\frac{1}{\sigma \sqrt{2 \pi}} \mathrm{e}^{-\frac{\epsilon^{2}}{2 \sigma^{2}}},-\infty<\epsilon<\infty, \sigma>0 .
$$

This is the derivation of the Gaussian or normal density given by Gauss, and hence it is also called the Gaussian density or error curve. Mathai and Pederzoli (1977) contains such characterizations of the normal probability law by using structural properties, regression properties etc. One fundamental idea was introduced in this area by Gordon and Mathai (1972). They tried to come up with pseudo-analytic functions of matrix argument involving rectangular matrices and by using this, characterization theorems were established for a multivariate normal density.

In Mathai and Rathie (1975), axiomatic definitions of information theory measures and basic statistical concepts are given. This is the first book giving axiomatic definitions of information measures. The techniques used are mainly from functional equations by using the proposed axioms create a functional equation and obtain its unique solution by imposing more conditions, if necessary, thus coming up with a unique definition or characterization of the concept. One such measure there is the one introduced as Havrda-Charvát measure, which for the continuous case is the following:

$$
H_{\alpha}(f)=\frac{\int_{-\infty}^{\infty}[f(x)]^{\alpha} \mathrm{d} x-1}{2^{1-\alpha}-1}, \alpha \neq 1
$$

where $f(x)$ is a density of the real scalar variable $x$. There is a corresponding discrete analogue, which is given by

$$
H_{\alpha}\left(p_{1}, \cdots, p_{k}\right)=\frac{\sum_{j=1}^{k}\left[p_{j}\right]^{\alpha}-1}{2^{1-\alpha}-1}, \alpha \neq 1
$$

where $p_{j}>0, j=1, \cdots, k ; p_{1}+\cdots+p_{k}=1$. A modified form of (9.2) and (9.3) is Tsallis entropy given by

$$
T_{q}(f)=\frac{\int_{-\infty}^{\infty}[f(x)]^{q} \mathrm{~d} x-1}{1-q}, q \neq 1
$$

for the continuous case, with a corresponding discrete analogue. Optimization of (9.4) under the condition that the total energy is preserved or the first moment is fixed, leads to Tsallis statistics of non-extensive statistical mechanics. Tsallis statistics is of the following form:

$$
p(x)=[1-(1-q) x]^{\frac{1}{1-q}}
$$

which is also a power law in the sense $\frac{\mathrm{d}}{\mathrm{d} x} p(x)=-[p(x)]^{q}$. Note that a direct optimization of (9.4), under the assumption that the first moment in $f(x)$ is fixed, does not yield (9.5) directly. One has to go through an escort density 


$$
g(x)=\frac{[p(x)]^{q}}{\int_{-\infty}^{\infty}[p(x)]^{q} \mathrm{~d} x}
$$

and then assume that the first moment is fixed in the escort density $g(x)$, to get the form in (9.5). Mathai's entropy

$$
M_{q}(f)=\frac{\int_{x}[f(x)]^{\frac{\eta+1-q}{\eta}} \mathrm{d} x-1}{q-1}, q \neq 1, q<1+\eta, \eta>0
$$

when optimized under the condition of first moment in $f(x)$ being fixed leads to Tsallis statistics directly. Also the optimization of (9.6) under two moment-type conditions leads to the pathway model, discussed in Section 3, where (9.5) will be a particular case.

\section{Biological Modeling: Formation of Pattern}

The most significant contribution in this area is the proposal of a theory of growth and form in nature and the explanation of the emergence of beautiful patterns in sunflower, along with explanation for the appearance of Fibonacci sequence and golden ratio there. The mathematical reconstruction of the sunflower head, with all the features that are seen in nature, is still the cover design of the journal of Mathematical Biosciences. The paper of Mathai and Davis appeared in that journal in 1974 and in 1976 the journal adopted the mathematically reconstructed sunflower head of Mathai and Davis (1974) as the journal's cover design with acknowledgement to the authors. When this paper was sent for publication to this journal, the editor wrote back saying: "enthusiastically accepted for publication" because this was the first time all natural features were explained in full. As per Davis and Mathai, the programming of the sunflower head is like a point moving along an Archimedes' spiral at a constant speed so that when the point makes an angle $\theta$, a second point starts and moves at the same speed. When the second point comes to $\theta$, a third point starts, and so on. The rule governing the movement is $f\left(\theta_{1}\right)+f\left(\theta_{2}\right)=f\left(\theta_{1}+\theta_{2}\right)$ or $r=k \theta$ where $k$ is a constant, giving Archimedes' spiral. When $\theta \approx 137.5^{\circ}$ or

$\frac{\theta}{2 \pi-\theta}=\frac{\sqrt{5}-1}{2}=$ golden ratio, one obtains sunflower, coconut tree crown, certain cactus heads and so on. Such a movement can be generated by a viscous fluid flowing up through a capillary with valves so that when a certain pressure is built up in one chamber the liquid moves up to the next chamber. The continuous flow is made pulse-like at the end. The upward motion can be effected by an evaporation process in the leaves, and there is no need for a heart-like mechanism in trees, pumping the fluid up. Mathai and Davis (1973) showed that the arrangments of leaves on a coconut tree crown is ideal from many mathematical points of view.

\section{Design of Experiments and Analysis of Variance}

The first paper of Mathai (Mathai, 1965), was on an approximate analysis of va- 
riance. It was on the analysis of a two-way classification with multiple observations per cell. Here the orthogonality is lost, and when estimating the main effects, one ends up in a singular system of linear equations of the form

$$
(I-A) \alpha=b
$$

where $A=\left(a_{i j}\right), m \times m, a_{i j}=\frac{n_{i j}}{n_{i .}}, n_{i .}=\sum_{j=1}^{m} n_{i j}, n_{i j}>0$ for all $i$ and $j$, is called the incidence matrix and the sum of the elements in each row is equal to 1 . Thus $I-A$ is singular and hence one cannot write it as $\alpha=(I-A)^{-1} b$ where $A$ and $b$ are known and the $m \times 1$ vector $\alpha$ is unknown and is to be estimated. Mathai noted that one could profitably use the conditions in the design and make $I-A$ a nonsingular matrix. One condition in the design is that $\alpha_{1}+\cdots+\alpha_{m}=0$ where $\alpha_{j}, j=1, \cdots, m$ are the elements in $\alpha$. Let $C$ be a matrix where all elements in the $i$-th row of $C$ are the median of the $i$-th row elements in $A$, namely the median of $a_{i 1}, \cdots, a_{i m}$ for $i=1, \cdots, m$. Then evidently $\mathrm{C} \alpha=O \quad$ (null). Then

$$
(I-A) \alpha=b \Rightarrow(I-B+C) \alpha=b \Rightarrow(I-B) \alpha=b
$$

where $B=\left(b_{i j}\right), b_{i j}=a_{i j}-c_{i}$ and $c_{i}$ is the median of the $i$-th row elements in $A$. Then a norm of $B$ is $\|B\|=\max _{i} \sum_{j=1}^{m}\left|a_{i j}-c_{i}\right|$. But since the mean deviation from the median is the least, $\|B\|$ is the minimum under the circumstances. Therefore, not only that $I-B$ is nonsingular but the series $I+B+B^{2}+\cdots$ is the fastest converging series for the problem at hand. Then

$$
(I-B) \alpha=b \Rightarrow \alpha=\left(I+B+B^{2}+\cdots\right) b
$$

A good approximation for $\alpha$ is available as $\alpha \approx(I+B) b$. This is found to be sufficient for all practical purposes of testing of statistical hypotheses on the components of $\alpha$.

\section{Population Problems and Social Sciences}

A problem that was looked into was how to come up with a measure of "distance" or "closeness" or "affinity" between two sociological groups or how to say that one community is close to another community with respect to a given characteristic. Mathai introduced the concepts of "directed divergence", "affinity" etc from information theory to social statistics. Let

$P=\left(p_{1}, \cdots, p_{k}\right), p_{j}>0, j=1, \cdots, k, p_{1}+\cdots+p_{k}=1$ and $Q=\left(q_{1}, \cdots, q_{k}\right), q_{j}>0, j=1, \cdots, k, q_{1}+\cdots+q_{k}=1$ be two discrete populations. Consider the representation of $P$ and $Q$ as points on a hypersphere of radius 1 , $x_{1}^{2}+\cdots+x_{k}^{2}=1$. Then the points are $\left(\sqrt{p_{1}}, \cdots, \sqrt{p_{k}}\right)$ and $\left(\sqrt{q_{1}}, \cdots, \sqrt{q_{k}}\right)$. Consider $\cos \theta$ where $\theta$ is the angle between these vectors or points on the hypersphere. Note that the lengths of the vectors are

$$
\left\|\left(\sqrt{p_{1}}, \cdots, \sqrt{p_{k}}\right)\right\|=1,\left\|\left(\sqrt{q_{1}}, \cdots, \sqrt{q_{k}}\right)\right\|=1
$$

and hence 


$$
\cos \theta=\sum_{j=1}^{k} \sqrt{p_{j} q_{j}} .
$$

This is a measure of angular dispersion and it is usually called "affinity" between $P$ and $Q$ or Matusita's measure of affinity between two discrete distributions. George and Mathai computed "affinity" between communities with reference to the characteristic of production of children and found that the politicians' statements did not match with the realities. Thus, some politicians' claims of certain communities producing more children, was repudiated in a scientific way in George and Mathai (1974). They also studied the most important variable responsible for population growth, namely the interval between two live births in woman of child-bearing age group and proposed a model, George and Mathai (1975). They also gave a formula for estimating an event from information supplied by different agencies, replacing the popular Deming formula in this regard.

\section{Quadratic and Bilinear Forms}

Major contributions in these areas are summarized in the books Mathai and Provost (1992), and Mathai, Provost and Hayakawa (1995). There is a very important concept in quadratic forms in Gaussian random variables called chisquaredness of quadratic forms. That is, $X^{\prime} A X \sim \chi_{r}^{2}$ if and only if $A$ is idempotent and of rank $r$, where $X$ the $p \times 1$ vector having the standard normal distribution $N_{p}(O, I)$, that is, $X \sim N_{p}(O, I)$ and $\chi_{r}^{2}$ is a chisquare random variable with $r$ degrees of freedom. Is there a corresponding concept when dealing with bilinear forms? When the samples come from a bivariate Gaussian or normal population it is not difficult to work out the density of the sample correlation coefficient. But what about the density of the sample covariance, without the scaling factors of the standard deviations? Both these questions were answered by Mathai (1993c) where Mathai introduced a concept called Laplacianness of bilinear forms (Mathai, 1993b) and also worked out the density of the covariance structures observing that covariance structure is a bilinear form. The necessary and sufficient conditions for a bilinear form to be noncentral generalized Laplacian are given in Corollary 2.5.2 of Mathai, Provost and Hayakawa (1995). For a noncentral generalized Laplacian the moment generating function is of the form

$$
M(t)=\left(1-\beta^{2} t^{2}\right)^{-\alpha} \exp \left\{-2 \lambda+2 \lambda\left(1-\beta^{2} t^{2}\right)^{-1}\right\}
$$

where $\lambda$ is the non-centrality parameter.

\section{Reliability Analysis: Extension to Pathway Model and Matrix-Variate Case}

In the area of reliability analysis, the basic concepts are survival function, hazard function, cumulative hazard, system reliability, reliability in the presence of other variables such as covariates etc. In a series of papers, Mathai and Princy in 2016 introduced the pathway model into the area so that the desired shapes for 
hazard function and the desired reliability for systems with components in series and parallel architecture could be obtained by selecting appropriate models from the pathway family of functions. Then, these ideas were extended to situations where the input variable or the variable under consideration is a rectangular matrix. As a byproduct, Maxwell-Boltzmann distribution, Raleigh distribution, Dirichlet averages etc were extended to matrix-variate cases, see for example Mathai and Princy (2017a, 2017b).

\section{Mellin Convolutions of Products and Ratios and M-Convolutions}

Mellin convolutions of products and ratios involving two functions are available in the literature. Mathai illustrated how these concepts are connected to statistical distribution theory and fractional calculus. In fact, a general definition for fractional integrals is given by Mathai using Mellin convolutions of products and ratios involving two functions. Corresponding $\mathrm{M}$-convolutions involving two functions of matrix argument is Mathai's contribution. He has also given physical interpretations for M-convolutions as densities of symmetric products and symmetric ratios of matrices. Mathai also extended Mellin convolutions and M-convolutions to three or more functions, see Mathai (2018). When three functions are involved, one can obtain several integral representations for the same Mellin convolution and M-convolution. For example, consider the symmetric product of three $p \times p$ real symmetric positive definite matrices $X_{j}>O, j=1,2,3$. One can take symmetric products as $X_{1}^{\frac{1}{2}} X_{2}^{\frac{1}{2}} X_{3} X_{2}^{\frac{1}{2}} X_{1}^{\frac{1}{2}}$, $X_{1}^{\frac{1}{2}} X_{3}^{\frac{1}{2}} X_{2} X_{3}^{\frac{1}{2}} X_{1}^{\frac{1}{2}}, X_{2}^{\frac{1}{2}} X_{3}^{\frac{1}{2}} X_{1} X_{3}^{\frac{1}{2}} X_{2}^{\frac{1}{2}}$ etc. When the original densities are assumed to be functionally symmetric then all such symmetric forms will produce the same densities whereas each symmetric product produces an integral representation which will be all different. Thus, one gets a large number of different integral representations for the same density or M-convolution of a product. One can also obtain further representations by taking for example, $X_{1}^{\frac{1}{2}} Y X_{1}^{\frac{1}{2}}$, $Y=X_{2}^{\frac{1}{2}} X_{3} X_{2}^{\frac{1}{2}}$ and consider the original symmetric product of three matrices as symmetric products of two matrices each, which will produce several more integral representations. In the real scalar case the Mellin convolutions can be evaluated in terms of generalized special functions, thus producing integral representations for these special functions. For example, let $x_{j}, j=1,2,3$ be real scalar positive random variables, independently distributed and let $u=x_{1} x_{2} x_{3}$ the product.

$$
E\left[u^{s-1}\right]=E\left[x_{1}^{s-1}\right] E\left[x_{2}^{s-1}\right] E\left[x_{3}^{s-1}\right]=\psi(s)
$$

where $\psi(s)$ will be gamma products when $x_{j}$ 's have densities belonging to the pathway family of functions, namely type- 1 beta, type- 2 beta and gamma. Then the inverse of $\psi(s)$ is a G-function. Then this G-function has several 
different types of integral representations. A series of papers are written by Mathai by using these ideas connecting statistical distributions, fractional calculus, Mellin convolutions and M-convolutions, see for example Mathai (2017, 2018, 2019).

\section{Above Topics Itemized: New Concepts and Procedures}

The following are the new concepts, new ideas and new procedures introduced by Mathai (Figure 16):

- Developed "dispersion theory" in 1967;

- Developed a generalized partial fraction technique, with Rathie, in 1971;

- Developed an operator to evaluate residues when poles of all types of orders occur (1971);

- Introduced the phrases "statistical sciences" in 1971 thereby the phrase "mathematical sciences" came into existence;

- Proposed a theory of growth and forms in nature (1974), the theory still standed, mathematically reconstructed a sunflower head;

- Introduced the concepts of "affinity", "distance" etc. in social sciences and created a procedure to compare sociological groups (1974);

- Introduced functions of matrix argument through M-transforms and M-convolutions;

- Introduced a non-linear least square algorithm (1993);

- Solved Miles' conjecture in geometrical probabilities, created and solved parallel conjectures (1982);

- Introduced Jacobians of matrix transformations in solving problems of random volumes, replacing the complicated integral and differential geometry procedures (1982);

- Now meaningful physical interpretations are given for M-convolutions; Unique recovery of $f(X)$ from its M-transform is still a conjecture;

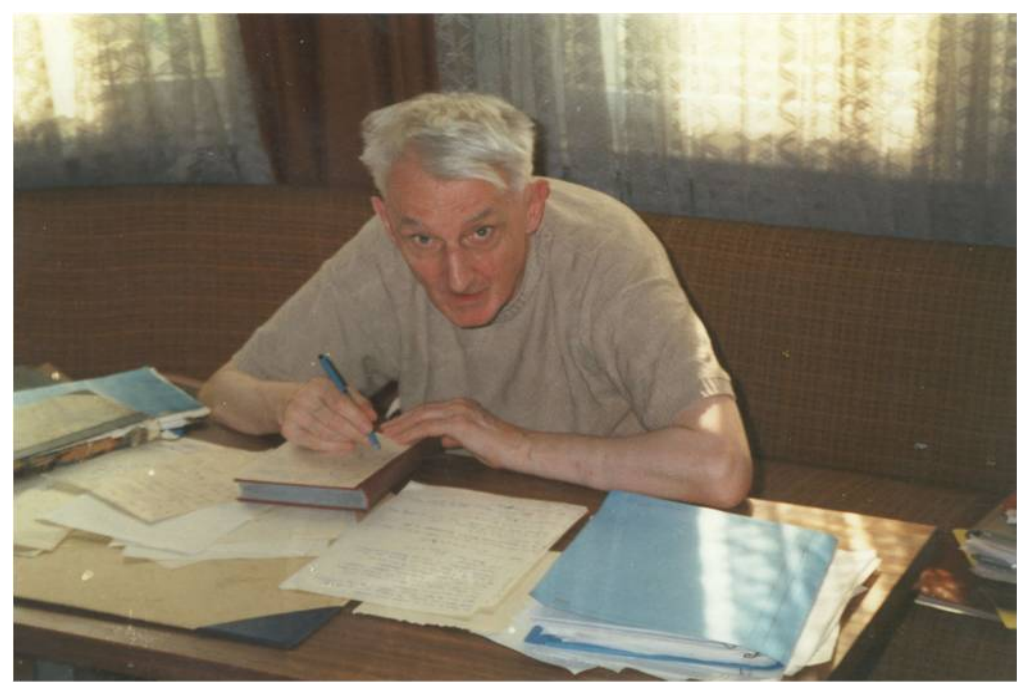

Figure 16. H.-J. Treder. 
- Extended Jacobians of matrix transformations from the real case to complex matrix-variate cases in a large number of situations;

- Introduced the concept of Laplacianness of bilinear forms and established the density of covariance structures (1993);

- Introduced pathway model and pathway idea (2005);

- Extended fractional calculus to real matrix-variate cases (2007);

- Established a connection between fractional calculus and statistical distribution theory (2007);

- Introduced Mathai's (2007) entropy;

- Geometrical interpretation and a general definition for fractional integrals were given (2013-2015);

- Extended fractional calculus to complex matrix-variate case and complex domain in general (2013);

- Extended fractional calculus to many matrix-variate cases (real and complex) (2014);

- Developed a fractional differential operator in the matrix-variate case (2015);

- Extended reliability analysis concepts to rectangular matrix-variate case (2017).

\section{Acknowledgements}

$\mathrm{HJH}$ expresses his deep appreciation for a lifelong support from and cooperation with Prof. Dr. A. M. Mathai, Department of Mathematics and Statistics, McGill University, Montreal, Canada, and Director of the Centre for Mathematical and Statistical Sciences, Peechi, Kerala, India. HJH also takes the opportunity to place on record his gratefulness for the encouragment of research by AkM Prof. Dr. Dr. e.h. mult. H.-J. Treder, Director of the Einstein Laboratory for Theoretical Physics, Caputh, Germany (see Schulz-Fieguth, 2018). Treder was the director of the Central Institute for Astrophysics of the Academy of Sciences (Berlin, GDR). In 1965 he was the principal organizer of the widely respected Einstein Symposium at the 50th anniversary of the invention of general relativity theory. For the Berlin international celebrations of Einstein's 100th birthday, 1979, he managed to secure the summer house of Einstein in Caputh, Brandenburg, as the Einstein Laboratory of Theoretical Physics in consultation with the administrators of the estate of Otto Nathan and Einstein. In 1981 he hosted the Michelson Colloquium at Potsdam to celebrate and recall the first Michelson experiment performed in 1881 at the Astrophysical Observatory in Potsdam. Treder was able to secure space and time for intense research work in his professional environment ranging from the solar neutrino problem (Treder, 1974) to fractional calculus (Treder, 1989). He supported actively the United Nations efforts to make available education and research in science to nations worldwide.

\section{Conflicts of Interest}

The authors declare no conflicts of interest regarding the publication of this paper. 


\section{References}

Beck, C. (2004). Superstatistics: Theory and Applications. Continuum Mechanics and Thermodynamics, 16, 293-304. https://doi.org/10.1007/s00161-003-0145-1

Cohen, E. G. D. (2004). Superstatistics. Physica D: Nonlinear Phenomena, 193, 35-52. https://doi.org/10.1016/j.physd.2004.01.007

Constantine, A. G. (1963). Some Noncentral Distribution Problems in Multivariate Analysis. Annals of Mathematical Statistics 34, 1270-1285. https://doi.org/10.1214/aoms/1177703863

Critchfield, C. L. (1972). Analytic Forms of the Thermonuclear Function. In F. Reines (Ed.), Cosmology, Fusion \& Other Matters: George Gamow Memorial Volume (pp. 186-191). Colorado: Colorado Associated University Press.

Davis Jr., R. (2003). A Half Century with Solar Neutrinos. Reviews of Modern Physics, 75, 985-994. https://doi.org/10.1103/RevModPhys.75.985

Fowler, A. W. (1984). Experimental and Theoretical Nuclear Astrophysics: The Quest for the Origin of the Elements. Reviews of Modern Physics, 56, 149-179.

https://doi.org/10.1103/RevModPhys.56.149

George, A., \& Mathai, A. M. (1974). Applications of the Concepts of Affinity and Distance to Population Problems. Journal of Biosocial Sciences (London), 6, 347-356. https://doi.org/10.1017/S0021932000009706

George, A., \& Mathai, A. M. (1975). Distribution of Inter-Live-Birth Intervals. Sankhya Series B, 37, 332-342.

Gordon, F. S., \& Mathai, A. M. (1972). Characterization of Multivariate Normal Distribution using Regression Properties. Annals of Mathematical Statistics, 43, 53-65. https://doi.org/10.1214/aoms/1177692714

Haubold, H. J., \& Mathai, A. M. (1988). Gravitational Instability in a Multicomponent Cosmological Medium. Journal of Mathematical Physics, 29, 2069-2077. https://doi.org/10.1063/1.527864

Haubold, H. J., \& Mathai, A. M. (1997). Sun. In J. H. Shirley, \& R. W. Fairbridge (Eds.), Encyclopaedia of Planetary Sciences (pp. 786-794). London: Chapman and Hall. https://doi.org/10.1007/1-4020-4520-4 390

Haubold, H. J., \& Mathai, A. M. (1998). Structure of the Universe,. In G. L. Trigg (Ed.), Encyclopedia of Applied Physics (pp. 47-51).

Haubold, H. J., \& Mathai, A. M. (Eds.) (2010). Proceedings of the Third UN/ESA/NASA Workshop on the International Heliophysical Year 2007 and Basic Space Science. Berlin and Heidelberg: Springer. https://doi.org/10.1007/978-3-642-03325-4

Haubold, H. J., Mathai, A. M., \& Saxena, R. K. (2011). Mittag-Leffler Functions and Their Applications. Journal of Applied Mathematics, 2011, Article ID: 298628. https://doi.org/10.1155/2011/298628

Herz, C. S. (1955). Bessel Functions of Matrix Argument. Annals of Mathematics, 63, 474-523.

James, A. T. (1961). Zonal Polynomials of the Real Positive Definite Symmetric Matrices. Annals of Mathematics, 74, 456-469. https://doi.org/10.2307/1970291

Kounias, S., \& Mathai, A. M. (1988). Maximizing the Sum of Integers When Their Sum of Squares Is Fixed. Optimization, 19, 123-131. https://doi.org/10.1080/02331938808843325

Krätzel, E. (1979). Integral Transforms the Bessel Type. In Generalized Functions \& Operational Calculus, Proc. Conf. Verna, 1975 (pp. 148-165). Sofia: Bulg. Acad. Sci. 
Mathai, A. M. (1965). An Approximate Analysis of a Two-Way Layout. Biometrics, 21, 376-385. https://doi.org/10.2307/2528097

Mathai, A. M. (1982). On a Conjecture in Geometric Probability Regarding Asymptotic Normality of a Random Simplex. Annals of Probability, 10, 247-251. https://doi.org/10.1214/aop/1176993929

Mathai, A. M. (1989). On a System of Differential Equations Connected with the Gravitational Instability in a Multi-Component Medium in Newtonian Cosmology. Studies in Applied Mathematics, 80, 75-93. https://doi.org/10.1002/sapm198980175

Mathai, A. M. (1993a). A Handbook of Generalized Special Function for Statistical and Physical Sciences. Oxford: Oxford University Press.

Mathai, A. M. (1993b). On Generalized Laplacianness of Bilinear Forms in Normal Variables. Journal of Multivariate Analysis, 45, 239-246.

https://doi.org/10.1006/jmva.1993.1036

Mathai, A. M. (1993c). The Residual Effect of a Growth-Decay Mechanism and the Distribution of Covariance Structures. Canadian Journal of Statistics, 21, 277-283. https://doi.org/10.2307/3315753

Mathai, A. M. (1997). Jacobians of Matrix Transformations and Functions of Matrix Argument. New York: World Scientific Publishing. https://doi.org/10.1142/3438

Mathai, A. M. (1998). Pollution by Vehicular Travels from the Suburbs to the City Core. Environmetrics, 9, 617-628. https://doi.org/10.1002/(SICI)1099-095X(199811/12)9:6<617::AID-ENV326>3.0.CO;2$\underline{E}$

Mathai, A. M. (1999a). An Introduction to Geometrical Probability: Distributional Aspects with Applications. Newark: Gordon and Breach.

Mathai, A. M. (1999b). Random p-Parallelotope in Euclidean n-Space. Advances in Applied Probability, 31, 343-354. https://doi.org/10.1239/aap/1029955138

Mathai, A. M. (1999c). Buffon Type Problem in the Euclidean Space R3. Rendiconti del Circolo Matematico di Palermo, XLVIII, 487-506. https://doi.org/10.1007/BF02844338

Mathai, A. M. (2003). Order Statistics from a Logistic Distribution and Applications to Survival \& Reliability Analysis. IEEE Transactions on Reliability, 52, 200-206. https://doi.org/10.1109/TR.2003.813432

Mathai, A. M. (2005). A Pathway to Matrix-Variate Gamma and Normal Densities. Linear Algebra and Its Applications, 396, 317-328. https://doi.org/10.1016/j.laa.2004.09.022

Mathai, A. M. (2007). Random Volumes under a General Matrix-Variate Model. Linear Algebra and its Applications, 425, 162-170. https://doi.org/10.1016/j.laa.2007.03.022

Mathai, A. M. (2009). Fractional Integrals in the Matrix-Variate Cases and Connection to Statistical Distributions. Integral Transforms and Special Functions, 20, 871-882. https://doi.org/10.1080/10652460902867791

Mathai, A. M. (2012). Generalized Krätzel Integral and Associated Statistical Densities. International Journal of Mathematical Analysis, 6, 2501-2510.

Mathai, A. M. (2013). Fractional Integral Operators in the Complex Matrix-Variate Case. Linear Algebra and its Applications, 439, 2901-2913. https://doi.org/10.1016/j.laa.2013.08.023

Mathai, A. M. (2014). Fractional Integral Operators Involving Many Matrix Variables. Linear Algebra and its Applications, 446, 196-215.

https://doi.org/10.1016/j.laa.2014.01.003 
Mathai, A. M. (2015). Fractional Differential Operators in the Complex Matrix-Variate Case. Linear Algebra and its Applications, 478, 200-217. https://doi.org/10.1016/j.laa.2015.03.038

Mathai, A. M. (2017). A Variant of Type-1 Beta and Dirichlet Integrals. Journal of the Ramanujan Society of Mathematics and Mathematical Sciences, 6, 25-40.

Mathai, A. M. (2018). Mellin Convolutions, Statistical Distributions and Farctional Calculus. Fractional Calculus \& Applied Analysis, 21, 376-398. https://doi.org/10.1515/fca-2018-0022

Mathai, A. M. (2019). Extended Fractional Integrals, Products and Ratios of Matrices and Statistical Distributions. 67th Annual Conference of Bharat Ganita Parishad, Lucknow, India, November 2019.

Mathai, A. M., \& Davis, T. A. (1973). A Mathematical Explanation of the Emergence of Foliar Spirals in Coconut and Other Palm Trees. Proceedings of the National Science Academy (India), 39, 194-202.

Mathai, A. M., \& Davis, T. A. (1974). Constructing a Sunflower Head. Mathematical Biosciences, 20, 117-133. https://doi.org/10.1016/0025-5564(74)90072-8

Mathai, A. M., \& Haubold, H. J. (1988). Modern Problems in Nuclear and Neutrino Astrophysics. Berlin: Akademie-Verlag.

Mathai, A. M., \& Haubold, H. J. (2008). Special Functions for Applied Scientists. New York: Springer. https://doi.org/10.1007/978-0-387-75894-7

Mathai, A. M., \& Haubold, H. J. (2017a). Linear Algebra for Physicists and Engineers. Berlin: De Gruyter. https://doi.org/10.1515/9783110562507

Mathai, A. M., \& Haubold, H. J. (2017b). Probability and Statistics for Physicists and Engineers. Berlin: De Gruyter. https://doi.org/10.1515/9783110562545

Mathai, A. M., \& Haubold, H. J. (2017c). Fractional and Multivariable Calculus: Model Building and Optimization Problems. Switzerland: Springer.

https://doi.org/10.1007/978-3-319-59993-9

Mathai, A. M., \& Haubold, H. J. (2018a). United Nations Basic Space Science Initiative (UNBSSI) 1991-2012 and beyond. Creative Education, 9, 192-248. https://doi.org/10.4236/ce.2018.92015

Mathai, A. M., \& Haubold, H. J. (2018b). Matrix Methods and Fractional Calculus. London: World Scientific Publishing. https://doi.org/10.1142/10639

Mathai, A. M., \& Haubold, H. J. (2018c). An Introduction to Fractional Calculus. New York: Nova Science Publishers. https://doi.org/10.1142/10639

Mathai, A. M., \& Haubold, H. J. (2018d). Erdélyi-Kober Fractional Calculus: From a Statistical Perspective, Inspired by Solar Neutrino Physics. In Springer Briefs in Mathematical Physics Volume 31. Singapore: Springer. https://doi.org/10.1007/978-981-13-1159-8

Mathai, A. M., \& Katiyar, R. S. (1979). Exact Percentage Points for Testing Independence. Biometrika, 66, 353-356. https://doi.org/10.1093/biomet/66.2.353

Mathai, A. M., \& Katiyar, R. S. (1993). A New Algorithm for Nonlinear Least Squares. Researches in Mathematical Statistics (Russian), 207, 143-157. (Translation by the American Mathematical Society)

Mathai, A. M., \& Moschopoulos, P. G. (1999). Pollution by Vehicular Travels from Satellite Townships to the City. Environmetrics, 10, 791-802.

https://doi.org/10.1002/(SICI)1099-095X(199911/12)10:6<791::AID-ENV396>3.0.CO;2 $\underline{-\mathrm{R}}$ 
Mathai, A. M., \& Pederzoli, G. (1977). Characterization of the Normal Probability Law. New Delhi: Wiley Eastern; and New York: Wiley Halsted.

Mathai, A. M., \& Princy, T. (2017a). Maxwell-Boltzmann and Rayleigh Distributions for the Rectangular Matrix-Variate Case. Physica A, 532, 287-311.

Mathai, A. M., \& Princy, T. (2017b). Analogues of Reliability Analysis for the Matrix-Variate Case. Linear Algebra and Its Applications, 532, 287-311. https://doi.org/10.1016/j.laa.2017.06.022

Mathai, A. M., \& Provost, S. B. (1992). Quadratic Forms in Random Variables: Theory and Applications. New York: Marcel Dekker.

Mathai, A. M., \& Provost, S. B. (2005). Some Complex Matrix-Variate Statistical Distributions on Rectangular Matrices. Linear Algebra and its Applications, 410, 198-216. https://doi.org/10.1016/j.laa.2005.07.016

Mathai, A. M., \& Provost, S. B. (2006). On q-Logistic and Related Distributions. IEEE Transactions on Reliability, 55, 237-244. https://doi.org/10.1109/TR.2006.874927

Mathai, A. M., \& Rathie, P. N. (1971). Exact Distribution of Wilks' Criterion. Annals of Mathematical Statistics, 42, 1010-1019. https://doi.org/10.1214/aoms/1177693329

Mathai, A. M., \& Rathie, P. N. (1972). Enumeration of Almost Cubic Maps. Journal of Combinatorial Theory, 13, 83-90. https://doi.org/10.1016/0095-8956(72)90012-3

Mathai, A. M., \& Rathie, P. N. (1975). Basic Concepts in Information Theory and Statistics: Axiomatic Foundations and Application. New York: Wiley Halsted; and New Delhi: Wiley Eastern.

Mathai, A. M., \& Saxena, R. K. (1969). Distribution of a Product and Structural Setup of Densities. Annals of Mathematical Statistics, 40, 1439-1448.

https://doi.org/10.1214/aoms/1177697515

Mathai, A. M., \& Saxena, R. K. (1973). Generalized Hypergeometric Functions with Applications in Statistics and Physical Sciences. Heidelberg: Springer. https://doi.org/10.1007/BFb0060468

Mathai, A. M., \& Saxena, R. K. (1978). The H-Function with Applications in Statistics and Other Disciplines. New York: Wiley Halsted; and New Delhi: Wiley Eastern.

Mathai, A. M., Provost, S. B., \& Hayakawa, T. (1995). Bilinear Forms and Zonal Polynomials. In Lecture Notes in Statistics 102. New York: Springer. https://doi.org/10.1007/978-1-4612-4242-0

Mathai, A. M., Saxena, R. K., \& Haubold, H. J. (2010). The H-Function: Theory and Applications. New York: Springer. https://doi.org/10.1007/978-1-4419-0916-9

Pyenson, L., Mathai, A. M., \& Haubold, H. J. (2019). United Nations Education Program in Space Science and Technology 1988-2018. Creative Education, 10, 2219-2231. https://doi.org/10.4236/ce.2019.1010160

Sakurai, K. (2014). Solar Neutrino Problems-How They Were Solved. Tokyo: Terrapub.

Schulz-Fieguth, M. (2018). Hans-Jürgen Treder-Ein Porträt. Potsdam, Germany: Christian and Cornelius Rüss.

Thomas, S., \& Mathai, A. M. (2009). P-Content of p-Parallelotope and Its Connection to Likelihood Ratio Statistics. Sankhya Series A, 71, 49-63.

Treder, H.-J. (1974). Gravitation und weitreichende schwache Wechselwirkungen bei Neutrino-Feldern (Gedanken zu einer Theorie der solaren Neutrinos). Astronomische Nachrichten, 295, 169-184. https://doi.org/10.1002/asna.19742950403

Treder, H.-J. (1989). Fraktale und gebrochene Ableitungen. Annalen der Physik, 46, 74-75. https://doi.org/10.1002/andp.19895010110 